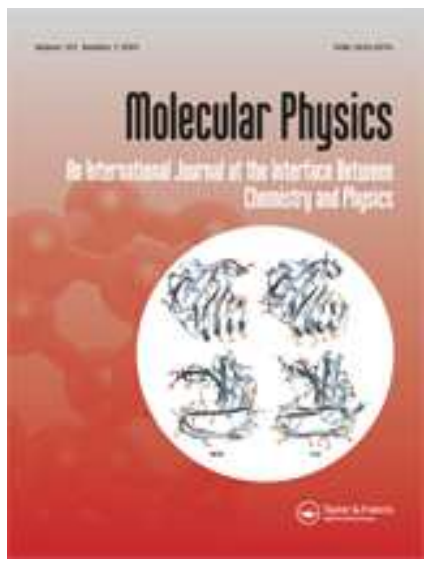

\title{
Renner-Teller Interaction, High Angular Momentum States and Spin-Orbit Interaction in the Electronic Spectrum of ND2
}

\begin{tabular}{|c|c|}
\hline Journal: & Molecular Physics \\
\hline Manuscript ID: & TMPH-2007-0056.R1 \\
\hline Manuscript Type: & Full Paper \\
\hline $\begin{array}{l}\text { Date Submitted by the } \\
\text { Author: }\end{array}$ & 29-Mar-2007 \\
\hline Complete List of Authors: & $\begin{array}{l}\text { Duxbury, Geoffrey; HOME; University of Strathclyde, Physics } \\
\text { Reid, Jonathan; University of Bristol, School of Chemistry }\end{array}$ \\
\hline Keywords: & $\begin{array}{l}\text { Electronic spectra, Vibronic coupling, Spin-orbit coupling, Renner- } \\
\text { Teller effect }\end{array}$ \\
\hline
\end{tabular}

\section{s ScholarONE" \\ Manuscript Central}


Renner-Teller Interaction, High Angular Momentum States and Spin-Orbit Interaction in the Electronic Spectrum of $\mathrm{ND}_{2}$

Geoffrey Duxbury ${ }^{1}$ and Jonathan P Reid ${ }^{2}$

${ }^{1}$ Department of Physics, University Of Strathclyde, Glasgow G4 0NG, Scotland, United Kingdom

${ }^{2}$ School of Chemistry, University of Bristol, Bristol BS8 1TS

\begin{abstract}
A rotational and vibrational analysis has been made of the $\mathrm{ND}_{2} \tilde{A}^{2} A_{1}-\tilde{X}^{2} B_{1}$ emission spectrum produced from the ultraviolet laser induced dissociation of both jet cooled and room temperature deuterated ammonia. By a combination of these experimental data with model calculation based upon the stretch-bender Renner-Teller Hamiltonian, and a previous analysis of the $\mathrm{NH}_{2}$ spectrum, most of the strong features in the emission spectra have been assigned. The spectrum consists of transitions from a narrow distribution of high angular momentum states peaking at $\mathrm{K}_{\mathrm{a}}$ values of 7 in the jet condition, and 8 at room temperature. Information has also been gained on the high angular momentum states of previously inaccessible higher bending levels from $\mathrm{v}_{2}=2$ to $\mathrm{v}_{2}=6$ in the $\tilde{X}^{2} B_{1}$ state.
\end{abstract}

\title{
Introduction
}


The Born-Oppenheimer approximation ${ }^{1}$, published in 1927 , is used to allow the separation of the high frequency electronic motion from the low frequency nuclear motion, and hence the separation of the vibronic Hamiltonian into an electronic and a nuclear Hamiltonian. For this to be the case the vibrational energy separations must be at least two orders of magnitude smaller than the electronic energy separations. Similarly vibration and rotation may be separated if the rotational energy separations are at least two orders of magnitude smaller than the vibrational energy separations. In the period from 1927 until about 1960, much of the theoretical effort was devoted to methods of deriving information about potential energy curves of electronic states of diatomic molecules, or the potential energy surface of an electronic state of a polyatomic molecule directly from the analysis of a high resolution spectrum. In particular the analysis of the spectra recorded in the infrared and microwave region relied upon the separation of vibrational and rotational motion.

Within ten years of the Born-Oppenheimer paper ${ }^{1}$, Teller and his collaborators ${ }^{2,3}$ had identified specific examples of molecules in which the breakdown of the BornOppenheimer separation could occur. The first of these is the Renner (or RennerTeller) effect ${ }^{2}$ and the second is the Jahn-Teller effect ${ }^{3}$. Although the original RennerTeller paper, describing in detail the breakdown of the Born-Oppenheimer separation in linear open-shell molecules, was published by Renner in $1934^{2}$, the first experimental measurements of a molecular example, the spectrum of $\mathrm{NH}_{2}$ were not made until 1952 by Herzberg and Ramsay ${ }^{4}$, and a detailed analysis published by Dressler and Ramsay in $1959^{5}$. The spectrum of $\mathrm{NH}_{2}$ is not an example of the type of spectrum envisaged by Renner, as the static splitting between the two component states is very large, with the lower component state having a bent equilibrium 
configuration. A second molecule, NCO, whose absorption spectrum was first recorded in $1958^{6}$, and analysed by Dixon in $1960^{7}$ was the first molecule with a linear equilibrium configuration of the type which Renner $^{2}$ had considered. A theoretical treatment of the energy level pattern in $\mathrm{NH}_{2}$ was given by Pople and Longuet-Higgins $^{8}$ and of the combined Renner and spin-orbit coupling in NCO type molecules by Pople 9 .

In 1961 Longuet Higgins ${ }^{10}$ wrote a review about both the Renner-Teller and JahnTeller effect as two related examples of the strong coupling between vibrational and electronic motions of molecules. In his introduction he wrote that "Most of us are so used to thinking of the two independently that it requires quite an effort of mental adjustment to abandon the idea that vibrational motions are determined by uniquely defined potential functions." He also noted, referring implicitly to recent experimental studies of that era such as the analyses of the spectra of $\mathrm{NH}_{2}$ and of $\mathrm{NCO}$, that "coupling effects produce, in particular molecules, some striking experimental phenomena". More recently Peric and Peyerimhoff ${ }^{11}$ have given an excellent comprehensive survey of the status of the Renner-Teller effect forty years on. In their review they have classified the Hamiltonians used into "Minimal Models", "Pragmatic Models", Benchmark Handling" and "Effective Hamiltonians". It is the purpose of the present paper to show that the "Minimal Models" and "Pragmatic Models" constitute types of effective Hamiltonians, as do the "Effective Hamiltonians" defined by Peric and Peyerimhoff. The main differences between them are associated with the type of reference frame used to help simplify the calculation, and also the amplitude of the motion of the bending motion, which leads to the splitting of the electronic degeneracy. 
The vehicle which we have chosen the demonstrate the applicability and usefulness of the pragmatic approach to the development and use of such effective Hamiltonians is the recently recorded emission spectrum of $\mathrm{ND}_{2}{ }^{12}$, and the contrast between the type of problem posed by such a spectrum and the original $\mathrm{ND}_{2}$ spectrum analysed by Dressler and Ramsay ${ }^{5}$. We may note that in their original analysis of the spectrum of $\mathrm{NH}_{2}$, Dressler and Ramsay made great use of the "minimal" model of Pople and Longuet - Higgins $^{8}$ in order to understand the complex structure of the observed spectrum. In fact the comparison between the predicted and observed vibronic patterns given in their paper gave a very compelling verification of the occurrence of Renner-Teller coupling in the $\mathrm{NH}_{2}$ free radical.

In the $\mathrm{NH}_{2}$ free radical there is a very large change in the equilibrium bond angle between the ground state, whose geometry is very similar to that of water, and the excited state, which is almost linear. Both the ground $\tilde{X}^{2} B_{1}$ and the excited $\tilde{A}^{2} A_{1}$ state correlate with the two components of a degenerate ${ }^{2} \Pi_{u}$ state of linear $\mathrm{NH}_{2}$. The Renner-Teller coupling is therefore between two states with a large static splitting of their potential energy surface at equilibrium, but which may become very strongly dynamically- coupled when the molecule executes large amplitude bending motion. Since the equilibrium geometries are so different, the absorption spectrum structure is dominated by the Franck-Condon allowed transitions, which results in the probing of excited states where the molecule is executing large amplitude bending motion. An understanding of the structure of the resulting spectrum therefore demands an understanding of both Renner-Teller and spin-orbit interaction and large amplitude motion. 
The original approaches to understanding the effects of large amplitude bending motion on Renner-Teller coupling, from the point of view of the analysis of experimental spectra, started about 1970 . This coincided with an interest in the large amplitude bending motion in closed shell molecules of the type considered originally by Hougen, Bunker and Johns $(\mathrm{HBJ})^{13}$, and continued by Bunker and Jensen ${ }^{14}$ and their collaborators and more recently Tennyson and his group ${ }^{15}$. Water was the first well documented example where the vibration rotation effective Hamiltonian expressed as a power series expansion of the type classified by Watson ${ }^{16}$ converged very slowly, and hence a search was made for methods of treating the large amplitude motion explicitly.

In the spectroscopy of closed shell molecules in which large amplitude motion is encountered, two basic philosophies have been used to achieving the construction of a satisfactory Hamiltonian, either concentrate on the isolation of the behaviour associated with a rogue large-amplitude coordinate, or use a new type of Hamiltonian in which motion in all coordinates is treated as potentially of large amplitude. With experimental evidence from the Frank-Condon patterns in electronic spectra that vibronic transitions to highly excited bending levels could be observed, Hougen Bunker and Johns (HBJ) ${ }^{15}$ developed a new method based upon the use of an angular dependent reference frame. Their method treats the bending vibration as the rogue coordinate, while treating the rest of the motion using a standard effective Hamiltonian, as the stretching motion is assumed to be of small amplitude relative to the reference frame. The calculations of Tennyson and his group for triatomics ${ }^{15}$, and tetratomics ${ }^{11}$, are very difficult to carry out, and are typical of the use of an 
instantaneous axis Hamiltonian to solve the full three-dimensional vibration-rotation problem.

In open shell molecules the complexity of large amplitude motion on two coupled surfaces has meant that few groups have tackled the problem in the way in which Tennyson ${ }^{15}$ and his group have attacked the water problem. The closest to this has been the calculations of Carter and Handy ${ }^{17}$, using the ab initio data of Rosmus ${ }^{18}$ and Chambaud and their colleagues. In many triatomic molecules only the bending motion is of very large amplitude, but the coupling between the stretching and bending motion, whilst weak, can often lead to complicated vibrational resonances. Since experimental data of molecules such as $\mathrm{NH}_{2}$ may be partly analysed by experimentalists by treating the individual sub- bands in the spectrum as though they were similar in structure to those of diatomic molecules, it is indicative that the separation of the motion in the large amplitude coordinates from that of the rest of the vibration-rotation problem may be quite good as noted above. As a result one may seek to develop a Hamiltonian which is based upon the possibility of such a separation.

The first Hamiltonian of this type was developed by Dixon and his colleagues ${ }^{19}$, and was motivated by studies of the electronic spectrum of $\mathrm{PH}_{2}$, the phosphorous analogue of $\mathrm{NH}_{2} . \mathrm{PH}_{2}$, like $\mathrm{NH}_{2}$, has very different equilibrium geometries in the ground and first excited state. The method which they chose was to transform the coupled equations, including both the Renner-Teller and spin-orbit coupling, in order to produce rotationally adiabatic potential energy curves for the $\tilde{X}^{2} B_{1}$ and the $\tilde{A}^{2} A_{1}$ vibronic states at each value of the bending angle. The remaining Renner-Teller 
coupling between the $\tilde{X}^{2} B_{1}$ and the $\tilde{A}^{2} A_{1}$ states then entered owing to the noncommutation of the diagonal nuclear kinetic energy operator and the angle dependent coordinate transformation. This method was published by Barrow, Dixon and Duxbury in $1974^{19}$, and forms the basis of many of the methods subsequently used to tackle this type of problem. However, as there was little information about the location of the bending vibrational levels associated with the ground electronic state, a decision was taken to use a damping method $^{19}$ to remove the effect of strong resonant interactions between levels formally belonging to the $\tilde{X}^{2} B_{1}$ and the $\tilde{A}^{2} A_{1}$ states.

The key series of papers in which the effect of localised resonances were treated exactly were by Jungen and Merer and Hallin ${ }^{20-22}$. Their method was based on that of Renner $^{2}$, and transformed the Hamiltonian matrix rather than the interaction Hamiltonian. The relationship between this and the BDD method has been given by Duxbury and Dixon ${ }^{23}$. The usefulness of the original work on $\mathrm{NH}_{2}$ by Jungen, Hallin and Merer has been demonstrated many times over the years following its publication. For example, Vervloet ${ }^{23,24}$ was able to use their calculated energy levels to aid the analysis of the spectra of highly excited levels of the ground electronic state of $\mathrm{NH}_{2}$, whose details had not been included in their original fitting scheme. It therefore seems that this original bending-only model incorporates much of the essential physics of the problem, including the effect of the bonds stretching as the molecule executes very large amplitude bending motion. It was also used by Dixon and his colleagues ${ }^{26}$ as a starting point when they studied the states of high angular momentum in the electronic spectra of $\mathrm{NH}_{2}$ 
The rovibronic states of $\mathrm{ND}_{2}$ which we analyse in the present paper are also high angular momentum states which arise when the free radical is produced by the photodissociation of either $\mathrm{ND}_{3}$ or $\mathrm{ND}_{2} \mathrm{H}^{12}$. In these experiments the isotopic forms of the $\mathrm{NH}_{2}$ free radical have been studies to aid the understanding of the photodissociation processes which occur in vacuum ultraviolet photolysis of ammonia. The free radical is then studied either using a flow cell, or a supersonic jet. Studying the nascent states of the free radical allows part of the dissociation dynamics to be inferred. Much of the theoretical understanding of this has been due to Dixon ${ }^{26}$, and the experiments carried out by Dixon and Ashfold and their colleagues at Bristol $^{26,27}$, and Leone and his collaborators, particularly Woodbridge, Ashfold, Loomis and Reid at JILA ${ }^{28-30}$. The experimental data on $\mathrm{ND}_{2}$ analysed in this paper was recorded by Reid, Leone and Loomis at JILA ${ }^{12}$.

It might be wondered why these unusual states were not seen in the original flash photolysis experiments of Dressler and Ramsay. In the original flash photolysis method the trihydrides were photolysed in the presence of a high pressure of a noble gas such as argon to thermalise the free radical, so that its effective temperature was close to room temperature. As a result the absorption spectrum of $\mathrm{ND}_{2}$ recorded by Dressler and Ramsay ${ }^{5}$ contains only transitions with little excitation of rotational angular momentum about the linear molecule axis, $\mathrm{K}_{\mathrm{a}}$, whereas almost all the transitions which we have identified in emission have much higher values of $\mathrm{K}_{\mathrm{a}}$. There is therefore no overlap between the vibronic levels of the $\tilde{A}^{2} A_{1}$ state measured at JILA ${ }^{12}$ and those of the original experiments carried at the National Research Council of Canada in Ottawa. 


\section{Experimental}

A detailed description of the experimental apparatus has been given in previous publications on the dissociation dynamics of ammonia and deuterated ammonias, so only a very brief summary will be provided here ${ }^{12,28-30}$. The studies were performed both at room temperature, with a continuous flow of ammonia, and jet cooled, with a piezoelectric pulsed valve. The triggering of the ArF eximer laser, which initiates the photodissociation, and also the opening of the pulsed jet for the jet cooled studies, were both synchronised to the position of a moving mirror in a commercial continuous scan Fourier transform spectrometer (FTS). The emission from the electronically excited $\mathrm{ND}_{2}$ fragments was then recorded using the FTS in this gated mode, to give a time history of the fluorescence The spectrum was recorded in two wavenumber regions, from 5000 to $12,000 \mathrm{~cm}^{-1}$ using an InSb detector, from 10,000 to $15,000 \mathrm{~cm}^{-1}$ with a Si avalanche detector. In the overlap region from 10,000 to $12,000 \mathrm{~cm}^{-1}$ the signal to noise ratio of the spectra measured using the avalanche detector was usually better, and were mainly used for the spectroscopic analysis. The resolution of the emission spectra was $0.2 \mathrm{~cm}^{-1}$.

\section{Analysis of the $\mathrm{ND}_{2} \tilde{A}^{2} A_{1}-\tilde{X}^{2} B_{1}$ emission spectrum}

An analysis of much of the emission spectrum was made by Reid, Loomis and Leone ${ }^{12}$ in order to understand the effects of parent zero-point motion on the $\mathrm{ND}_{2}$ $\tilde{A}^{2} A_{1}$ rotational distribution. However in their paper only an outline of the rotational analysis was given. In the present paper we have extended the analysis by making use of stretch-bender ${ }^{31,32}$ calculations of the spectrum of $\mathrm{ND}_{2}$, using the potential 
developed by Alijah and Duxbury ${ }^{33,34}$ in their detailed modelling of the $\mathrm{NH}_{2}$ spectrum. We have also used the rotational term values of $\mathrm{ND}_{2}$ derived from the analysis of high resolution laser magnetic resonance, electronic spectra, and microwave optical double resonance, by Muenchausen et. al. ${ }^{35}$, and the very high angular momentum states measured in their far infrared FT spectra by Morino and Kawaguchi $^{36}$. Their use has been crucial in obtaining accurate values of the terms values in the $\tilde{A}^{2} A_{1}$ state, and subsequently of vibrationally excited levels in the $\tilde{X}^{2} B_{1}$ state.

Since the spectra of the $\mathrm{ND}_{2}$ free radicals are due to emission from nascent free radicals produced from the photodissociation of their parent deuterated ammonia, the population of the excited state energy levels from which emission is observed reflect the way in which the free radical is produced in the photodissociation process. The distribution of intensity in the emission spectrum therefore reflects the distribution of angular momentum about the least axis of inertia, the a-axis, which correlates with the axis of linearity of linear $\mathrm{ND}_{2}$. It has been found ${ }^{12}$ that in $\mathrm{ND}_{2}$ molecules produced by the photolysis of jet cooled $\mathrm{ND}_{3}$, the majority of the molecules are in rovibronic levels of the excited state with $\mathrm{K}_{\mathrm{a}}=7$. When room temperature $\mathrm{ND}_{3}$ is photolysed the levels with $\mathrm{K}_{\mathrm{a}}=8$ are also populated with a similar or slightly greater probability ${ }^{12}$ than those with $\mathrm{K}_{\mathrm{a}}=7$. The process responsible for this including the amount of available energy, has been described in a series of papers by Reid et al. ${ }^{12,29,30}$. In order to show the effect of the photodissociation to produce a very non-Boltzman population of the excited state levels, in Figure 1 a survey of the emission spectrum from the photolysis of jet cooled $\mathrm{ND}_{3}$ is shown. It may be seen that it is dominated by the ${ }^{\mathrm{R}} \mathrm{R}$ branches and ${ }^{\mathrm{P}} \mathrm{P}$ branches originating from the $\mathrm{K}^{\prime}=7$ angular momentum state of the zero-point 
vibrational level of the $\tilde{A}^{2} A_{1}$ state of $\mathrm{ND}_{2}$. In these low temperature spectra the main lines seen in these branches have $\mathrm{N}^{\prime}=\mathrm{K}_{\mathrm{a}}{ }^{\prime}=7$. The spectra obtained from the photolysis of room temperature $\mathrm{ND}_{3}$ are more complicated, but the origins of several of the branches with $\mathrm{K}_{\mathrm{a}}{ }^{\prime} \neq 7$ may be obtained by a direct comparison on these spectra with those obtained from the photolysis of the cold $\mathrm{ND}_{3}$. As only levels associated with the excitation of the bending vibration have been observed in these experiments, the transitions are labelled by the vibrational mode number, 2 , and the number of vibrational quanta of the bending vibration which are excited in the $\tilde{X}^{2} B_{1}$ and $\tilde{A}^{2} A_{1}$ states, i.e $2_{v^{\prime \prime}}^{v^{\prime}}$. The main bands seen in the cold spectrum are $2_{0}^{0}, 2_{1}^{0}, 2_{2}^{0}, 2_{3}^{0}, 2_{4}^{0}$ and $2_{5}^{0}$, as well as part of the $2_{6}^{0}$ band.

In the expanded section of the photodissociation product emission spectrum shown in Figure 2, it may be seen that in the room temperature dissociation spectrum there are a much larger number of emission lines than from the jet cooled parent. It may also be seen that the dominant pattern involves closely spaced doublets of approximately equal intensity. This doubling is due to spin-orbit spitting of the rovibronic energy levels in both the ground and the excited states of $\mathrm{ND}_{2}$. Using prolate symmetric rotor labelling with $\mathrm{K}=\mathrm{K}_{\mathrm{a}}$, and with $\mathrm{N}$ being the total angular momentum neglecting electron spin, the $\mathrm{F}_{1}$ level of the split rovibronic level has $\mathrm{J}=\mathrm{N}+1 / 2$ and the $\mathrm{F}_{2}$ level $\mathrm{J}=\mathrm{N}-1 / 2$. As all the doubling associated with a-axis rotation is related to the spinorbit splitting of the linear molecule, in the absence of strong localised resonances between the levels of the $\tilde{A}^{2} A_{1}$ and the $\tilde{X}^{2} B_{1}$ states, the sign of the splitting in the lower state is reversed from that in the excited state. It has been shown that in the $\tilde{X}^{2} B_{1}$ state $^{20-22}$ this splitting results in an inverted state where the energy of the $\mathrm{F}_{2}$ levels is greater than that of the $\mathrm{F}_{1}$ levels, whereas in the $\tilde{A}^{2} A_{1}$ levels identified in 
these photodissociation experiments the excited state levels are regular, with the energy of the $F_{1}$ levels greater than that of the $F_{2}$ levels. For the measured transitions between the zero point levels of both the ground and the excited states the spin-orbit splitting of the levels of the $\tilde{X}^{2} B_{1}$ state has been shown to be small, so that most of the spin-orbit splitting observed in the spectra shown in Figure 2 is due to that in the excited state. However, as the ${ }^{\mathrm{P}} \mathrm{P}, \mathrm{N}^{\prime}=\mathrm{N}^{\prime}+1, \mathrm{~K} "=8$. and the ${ }^{\mathrm{R}} \mathrm{R}, \mathrm{N}^{\prime}=\mathrm{N}^{\prime}-1, \mathrm{~K}=6$, both originate from the levels with $N^{\prime} \geq K^{\prime}, K^{\prime}=7$, the difference between the measured splitting of these transitions is related to the difference in the splitting in the $\tilde{X}^{2} B_{1}$ state. Moreover, as the energies of the $F_{1}$ and $F_{2}$ components of a rotational level in the ground state are inverted, with $F_{1}$ at lower energy than $F_{2}$, the magnitude of the splitting in these pairs of ${ }^{\mathrm{P}} \mathrm{P}$ and ${ }^{\mathrm{R}} \mathrm{R}$ branches is greater than that of the excited state $\mathrm{N}, \mathrm{K}$ level. We can make use of this when following the splitting of the equivalent branches of the sequence of levels terminating in successively higher bending vibrational levels of the ground electronic state. As a result we can investigate the influence on the magnitude of the spin-orbit splitting of these energy levels upon the decreasing separations of these energy levels from that of the zero-point level of the excited state. The variation of this splitting of equivalent transitions, having a common pair of $F_{1}$ and $F_{2}$ components with $K^{\prime}=7$, with low and high values of $v_{2}$ ", is shown in Figure 3.

The weaker emission lines seen in Figure 2 have two main origins, either they belong to perpendicular $\mathrm{Q}$ branches associated with the much stronger ${ }^{\mathrm{P}} \mathrm{P}$ and ${ }^{\mathrm{R}} \mathrm{R}$ branches, or they originate from excited bending levels of the $\tilde{A}^{2} A_{1}$ state. Reid et al ${ }^{30}$ have used a cross- correlation technique to analyse the change in the raw spectral emission data with the variation in mixture composition, as it was found that the emission spectra 
produced by the photodissociation on $\mathrm{ND}_{3}$ and $\mathrm{ND}_{2} \mathrm{H}$ are significantly different. In particular they used this method to identify which of the patterns seen in this spectral region originated from vibrationally excited levels of $\mathrm{ND}_{2}$ and which did not. An example is shown in Figure 5 of their paper. In Figure 4 we show an expanded part of the spectrum from room temperature dissociation, in which the ${ }^{\mathrm{R}} \mathrm{Q}$ branches which lie to the red of the associated ${ }^{\mathrm{R}} \mathrm{R}$ branch may be clearly seen. In Figure 5 we show an extract of a spectrum produced by the photodissociation of $\mathrm{ND}_{3}$, and one containing only emission lines from excited bending levels of $\mathrm{ND}_{2}$ derived by Reid et $\mathrm{al}^{30}$. Use of this spectrum has enabled us to clearly identify many of the remaining weak emission lines seen in the spectra obtained by the photodissociation of $\mathrm{ND}_{3}$.

The assignments of the emission spectra of $\mathrm{ND}_{2}$ are given in Table 1 . In Table 2 we give the excited state term values derived from the experimental measurements of transitions in the $2_{0}^{0}$ and $2_{1}^{0}$ bands by using the $\mathrm{v}_{2}=0$ and 1 term values obtained from the work of Muenchausen et. al. ${ }^{35}$ and Morino and Kawaguchi ${ }^{36}$. In this table they are compared with theoretical calculations based upon the $\mathrm{NH}_{2}$ spectrum ${ }^{33,34}$, using the stretch-bender Renner-Teller Hamiltonian ${ }^{32}$. In Table 3 we show the ground state term values derived from the earlier experimental work, and in Table 4 the term values of levels of the higher vibrational states of the ground electronic state. These latter term values were calculating by subtracting the values of the wavenumbers of the fluorescence spectra from the term values given in Table 2. The fluorescence spectra used were from the $2_{2}^{0}, 2_{3}^{0}, 2_{4}^{0}$ and $2_{5}^{0}$ bands, and also the much weaker $2_{6}^{0}$ band. 


\section{Stretch-Bender vibronic and rovibronic energy level calculations.}

The calculations of the structure of the spectrum of the high angular momentum transitions in $\mathrm{ND}_{2}$ was carried out using the Renner-Teller code based on the $\mathrm{BDD}^{19}$ Hamiltonian transformation coupled with the stretch-bender implementation ${ }^{31}$ of large amplitude bending and coupling with the symmetric stretching vibrations. The basis of this is described in detail by Duxbury et. al. ${ }^{31}$ and by Alijah and Duxbury ${ }^{32}$. The stretch-bender part of the Hamiltonian originated from an idea of Jungen, who together with Palivan developed its use for linear molecules, as well as for $\mathrm{CH}_{2}{ }^{31}$. In the present paper we have made use of the parameterisation used by Alijah and Duxbury $^{32-34}$ to fit the extensive data on the ground and excited states of $\mathrm{NH}_{2}$. The parameters used in the present calculations are given in Table 5. They are identical to those used by Duxbury and Alijah ${ }^{32-34}$ except for changes in the values of the asymmetric stretching frequencies in the ground and excited states, which are corrected for the effects of deuteration, and for an adjustment to the value of the parameter used to allow for the quenching of orbital angular momentum, $\mathrm{g}_{\mathrm{K}}$. In our treatment of the Renner-Teller coupling we have considered the quenching of the orbital angular moment as the molecule bends from the linear configuration. Following Jungen and Merer $^{20}$ we write the following expression for the electronic angular momentum coupling (an electronic Coriolis interaction) between the $\tilde{A}^{2} A_{1}$ and the $\tilde{X}^{2} B_{1}$ states:

$$
\hbar^{-1}\left\langle{ }^{e} A_{1}\left|L_{z}\right|{ }^{e} B_{1}\right\rangle=\zeta_{e}^{z} \equiv \Lambda(\rho)=\Lambda-u(\rho)
$$


In equation (1) $u(\rho)$ as a positive quantity, so that if it is non-zero its increase with increasing bending angle supplement, $\rho$, represent a quenching of angular momentum with departure from linearity. The function $g_{K}(\rho)$ was then defined ${ }^{20}$ as

$$
g_{K}(\rho)=2 A(\rho) u(\rho)=g_{K}^{0}+g_{K}^{2} \rho^{2}+\cdots
$$

where $A(\rho)$ is the angular dependent a-axis rotation constant, whose value tends to infinity as $\rho \rightarrow 0$. Hence, although $u(\rho)$ is isotopically independent, the value of $g_{K}^{0}$ is isotopically dependent, since the value of $A(\rho)$ for $\mathrm{ND}_{2}$ will be smaller than that of $\mathrm{NH}_{2}$ by a factor of $\sim 0.56^{31}$. This correction has been made to the value of $\mathrm{g}_{\mathrm{K}}$ to ensure isotopic invariance of the angular momentum quenching.

In Table 6 we show the values of the vibronic origins and effective spin-orbit coupling parameters, $A_{v_{2}, K}^{S O}$ of the vibronic levels observed in the original measurements by Dressler and Ramsay ${ }^{5}$, and in Table 7 the vibronic origins and $A_{v_{2}, K}^{S O}$ values for the high angular momentum states analysed in this work. It may be seen that the stretch-bender model using the $\mathrm{NH}_{2}$ potential parameters gives a good representation of the vibronic structure of the $\mathrm{ND}_{2}$ spectrum, and allows they results of the spectral analysis to be confirmed. The values of $A_{v_{2}, K}^{S O}$ in most of the excited state energy levels listed by Dressler and Ramsay ${ }^{5}$ are very small, owing the complicated mixing associated with strong Fermi resonance coupling as discussed by Alijah and Duxbury for $\mathrm{NH}_{2}{ }^{32-34}$. However the predicted behaviour of the splitting of levels with $K^{\prime}=1$ is well reproduced by the current calculations, including the prediction of whether the excited state levels are regular, $T_{N+1 / 2}>T_{N-1 / 2}$, or inverted, 
$T_{N+1 / 2}<T_{N-1 / 2}$. Since the splitting in the lowest vibrational levels of the $\tilde{X}^{2} B_{1}$ state is known to be inverted, the sign of the splitting in excited state levels may be inferred if the excited state term values are derived from two transition terminating in ground state levels with very different spin-rotation splittings. The term values derived by Dressler and Ramsay had taken this into account ${ }^{5}$, but there is no comment about it within their paper. The portion of the spectrum of the $20^{5}$ band (in the linear molecule labelling used by Dressler and Ramsay the excited state has $v_{2}=12$ ) shown in their paper shows clearly the spin-splittings in the $\Pi$ sub-band. It is the excited state levels of this transition which have an inverted spin-orbit splitting.

This behaviour contrasts with that of the spin-orbit splitting of the high angular momentum states of the transitions from the $\mathrm{v}_{2}=0$ and 1 states of $\tilde{A}^{2} A_{1}$ where the splitting is large and regular, as shown in Tables 2 and 7. It may be seen that the calculated values of the rotational dependence of the spin-orbit splittings presented in Table 2, which use the parameters derived from the least squares fitting by Alijah and Duxbury of the $\mathrm{NH}_{2}$ spectrum ${ }^{33,34}$, give a very good fit to the observed rotational variation of the spin-orbit splittings of the observed $\mathrm{ND}_{2}$ term values.

The small systematic shifts of the origin between the observed and calculated positions of the vibronic origins are probably due in part to the omission of some of the effects of the changes in zero point motion between the hydride and the deuteride. For the high angular momentum states the differences may also reflect the effects of the parameterisation of the bond stretching in the stretch-bender model, since the shape of the effective K-dependent bending potential energy curves discussed by Duxbury and Alijah ${ }^{32-34}$ is very dependent upon the effective bond length used to 
calculate $A(\rho)$, the angular dependent a-axis rotation constant. The variation of this effective potential with $\mathrm{K}$ is shown in Figure 8 of Ref. [34].

\section{Conclusion}

We have demonstrated that, even in the absence of ab-initio calculations, the type of effective Hamiltonian represented by the stretch-bender, with Renner-Teller and spinorbit coupling included, allows us to make connections between sets of experimental data recorded under very different conditions, and having almost no energy levels in common. As Peric and Peyerimhoff ${ }^{11}$ have noted, both the stretch-bender Hamiltonian $^{31,32}$ and the MORBID Hamiltonian ${ }^{14}$ of Jensen and Bunker, may give results which approach those of their "benchmark" goal. Most spectroscopic analysis has made use of some type of effective Hamiltonian to derive molecular information directly from the experimental spectrum. In studies of diatomic molecules much of the effort that has been made to derive as much information as possible directly from a spectrum has been described in detail by Lefebvre-Brion and Field ${ }^{37}$.

Even with the use of theoretical approaches of the type described by Jungen and Merer $^{20}$, and by Dixon and Duxbury ${ }^{19,23}$ and their collaborators, a direct attack on a spectrum requires high quality experimental data. The present analysis has certainly benefited from the availability of high quality spectroscopic information on the $0,0,0$ and $0,1,0$ levels of the ground $\tilde{X}^{2} B_{1}$ state, which have allowed us to derive the term values of rotational levels in the zero-point level excited $\tilde{A}^{2} A_{1}$ state having $\mathrm{K}_{\mathrm{a}}=6,7,8$ 
and 9. These levels have in turn been used to derive term values for rotational levels of the vibrational levels in the $\tilde{X}^{2} B_{1}$ state having values of $\mathrm{v}_{2}$ from 2 to 6 .

However the fact that only a limited number of vibronic states were accessed in the present series of experiments demonstrates a major problem in inverting the experimental spectra of polyatomic molecules to derive potential energy surfaces. The limitations on the states accessed are due to both the method of production of free radicals or radical ions, and also to the selectivity about which parts of the potential energy surface are explored due to the Franck-Condon factors. The major influence of the Franck-Condon principle on selectivity is exemplified by the electronic spectra of $\mathrm{NH}_{2}$ and $\mathrm{ND}_{2}$, where there is a great deal of information about bending but little about stretching, as there is very little change in bond-length on electronic excitation. This means that in general only a limited amount of experimental information about potential energy surfaces is available from the analysis of the spectra of polyatomic molecules, even those like $\mathrm{ND}_{2}$ having only one atom more than a diatomic molecule.

\section{Acknowledgements}

The experimental measurements were carried out at JILA by the group of S.R. Leone. We would like to thank him and his colleague R.A. Loomis for permission to use their data. Both of us have benefited from discussions with both S.R. Leone and R.A. Loomis in JILA at earlier stages of this analysis. We are also very grateful to Alex Alijah for all his help in developing the stretch-bender Renner-Teller model and computer code used here. 
References

$1 \quad$ M. Born and J.R. Oppenheimer, Ann. d. Physik. 87, 457 (1927)

$2 \quad$ R. Renner, Z. Phys., 92, 172 (1934)

3 H.A Jahn and E. Teller, Proc. Roy. Soc 161A, 220 (1937

4 G. Herzberg and D.A. Ramsay, J. Chem. Phys,. 20, 347 (1952)

$5 \quad$ K. Dressler and D.A. Ramsay Phil. Trans. Roy. Soc. 251A, 553 (1959)

6 R.Holland, D.W.G. Style, R.N. Dixon and D.A. Ramsay, Nature, 182, 336, (1958)

7 R.N. Dixon, Phil. Trans. Roy. Soc. 252A, 165 (1960)

8 J.A. Pople and H.C. Longuet Higgins, Molec. Phys. 1, 372 (1958)

9 J.A. Pople, Molec. Phys. 3, 16 (1960)

10 H.C. Longuet Higgins, Adv. Spectrosc., 2, 429 (1961)

11 M. Peric and S.D. Peyerimhoff. In Advances in Chemical Physics, I.

Prigogine and S.A. Rice Editors, John Wiley, New York 2002, Vol. 124, p. 583

12 J.P. Reid, R.A. Loomis and S.R Leone, Chem. Phys. Lett, 324 240248(2000)

13 J.T. Hougen, P.R. Bunker and J.W.C. Johns, J. Mol. Spectrosc., 34, 136 (1970)

14 P. Jensen, M. Brumm, W.P. Kraemer and P.R. Bunker, J. Mol. Spectrosc. 171. $31(1995)$

15 P Barletta, S.V. Shirin, N.F. Zobov, O.L. Polyansky, J. Tennyson, E.F. Valeev and A.G. Császar, J. Chem. Phys. 125, 204307 (2006)

16 J.K.G. Watson, in "Vibrational Spectra and Structure", Ed J.R. Durig, Vol 6 ,p. 1, Elsevier, Amsterdam (1982) 
17 S.Carter, N.C. Handy, P. Rosmus and G. Chambaud, Molec. Phys. 71,605 (1990)

18 W. Gabriel, G. Chambaud, P. Rosmus, S. Carter and N.C. Handy, Molec. Phys., 81, 1445 (1994)

19 T. Barrow, R.N. Dixon and G. Duxbury, Molec. Phys. 27, 1217 (1974)

20 Ch. Jungen and A.J. Merer, Molec. Phys., 40, 1 (1980)

21 Ch. Jungen, K-E. J. Hallin and A.J. Merer Molec. Phys., 40, 25 (1980)

22 Ch. Jungen, K-E. J. Hallin and A.J. Merer Molec. Phys., 40, 65 (1980)

23 G. Duxbury and R.N. Dixon, Molec. Phys., 43, 255, (1981)

24 M. Vervloet, Molec. Phys. 63, 43 (1988)

25 I. Hadj-Bachir, T.R. Huet, J.L. Destombes and M. Vervloet, J. Mol. Spectrosc, 193, 326 (199)

26 R.N. Dixon, S.J. Irving, J.R. Nightingale and M. Vervloet, J. Chem. Soc. Faraday Trans. 87, 2121-2133 (1991)

27 D.H. Mordaunt, M.N.R. Ashfold, and R.N. Dixon, J. Chem. Phys. 104, 6460-6471 (1996)

28 E.L. Woodbridge, , M.N.R. Ashfold, and S.R. Leone, J. Chem. Phys., 94, 4195-4204 (1991)

29 J.P. Reid, R.A. Loomis and S.R Leone, J. Phys. Chem. A, 10410139$10149(2000)$

30 J.P. Reid, R.A. Loomis and S.R Leone, J. Chem. Phys,. 112, 3181-3191 (2000)

31 G. Duxbury, A. Alijah, B.D. McDonald, M. Van Gogh, A. Alijah, Ch. Jungen and H. Palivan, J. Chem. Phys. 108, 2336 (1998)

32 A. Alijah and G. Duxbury, J. Mol Spectrosc. 211, 7-15 (2002) 
33 A. Alijah and G. Duxbury, J. Mol Spectrosc. 211, 16-30 (2002)

34 G. Duxbury and A. Alijah, J. Mol Spectrosc. 211, 31-57 (2002)

35 R.E, Muenchausen, G.W. Hills, M.F. Merienne-Lafore, D.A. Ramsay, M.

Vervloet and F.W. Birss. J. Mol. Spectrosc. 112, 203-210 (1985)

36 I. Morino and K. Kawaguchi, J. Mol. Spectrosc, 182, 428 (1997)

37 H. Lefebvre-Brion and R.W. Field, "The Spectra and Dynamics of

Diatomic Molecules", Elsevier Academic Press, Amsterdam, (2004) 
Table 1

Assignments of the emission spectra of $\mathrm{ND}_{2}$ recorded in time gated mode following the photo-dissociation of $\mathrm{ND}_{3}$. The sub branches are aligned so that transitions from lower state levels to a common upper state level are paired. The $\mathrm{K}$ values of the common upper state levels are indicated. The vibrational transitions are indicated using the notation (vibration number) $)_{\mathrm{v}^{\prime}}^{\mathrm{v}^{\prime}}$.

\begin{tabular}{|c|c|c|c|c|c|c|}
\hline${ }^{\mathrm{P}} \mathrm{P}$ branches & & & $\begin{array}{l}\text { Vibrational } \\
\text { Transition }\end{array}$ & ${ }^{\mathrm{R}} \mathrm{R}$ branches & & \\
\hline $\mathrm{F}_{1}$ & $\mathrm{~F}_{2}$ & & $2_{0}^{1}$ & $\mathrm{~F}_{1}$ & $\mathrm{~F}_{2}$ & \\
\hline 13691.91 & 13682.06 & P 8,8 & $\mathrm{~K}^{\prime}=7$ & 14061.85 & 14052.22 & R 6,6 \\
\hline 13666.67 & 13657.78 & P 9,8 & & 14058.89 & 14050.32 & R 7,6 \\
\hline 13640.00 & 13632.70 & P 10,8 & & 14054.07 & 14046.67 & R 8,6 \\
\hline 13390.74 & 13379.26 & P 7,7 & $\mathrm{K}^{\prime}=6$ & 13712.59 & 13701.48 & R 5,5 \\
\hline 13366.30 & 13357.78 & P 8,7 & & 13710.00 & 13701.48 & R 5,5 \\
\hline 13341.11 & 13333.7 & P 9,7 & & 13706.32 & 13699.63 & R 5,5 \\
\hline 13093.70 & 13084.63 & P 6,6 & $\mathrm{K}^{\prime}=5$ & 13365.42 & 13356.85 & $\mathrm{R} 4,4$ \\
\hline 12983.71 & 12976.91 & P 10,10 & $\mathrm{~K}^{\prime}=9$ & 13446.37 & 13439.78 & R 8,8 \\
\hline 12956.16 & 12948.73 & P 11,10 & & 13440.74 & 13434.07 & R 9,8 \\
\hline 12925.56 & 12918.89 & P 12,10 & & 13432.59 & 13425.93 & R 9,8 \\
\hline 12707.63 & 12699.02 & $\mathrm{P} 9,9$ & $\mathrm{~K}^{\prime}=8$ & 13124.46 & 13116.24 & R 7,7 \\
\hline 12679.84 & 12672.02 & P10,9 & & 13118.98 & 13111.55 & R 8,7 \\
\hline 12649.71 & 12643.44 & P11,9 & & 13110.96 & 13104.67 & R 9,7 \\
\hline 12432.68 & 12424.46 & P 8,8 & $\mathrm{~K}^{\prime}=7$ & 12802.94 & 12794.32 & R 6,6 \\
\hline 12406.26 & 12399.22 & P 9,8 & & 12798.24 & 12791.19 & R 7,6 \\
\hline 12378.67 & 12372.21 & P 10,8 & & 12792.68 & 12786.5 & R 8,6 \\
\hline
\end{tabular}


12164.19

$12156.75 \quad$ P 7,7

$K^{\prime}=6$

12486.11

12478.47 R 5,5

12534.83

$12524.67 \quad$ P 8,8

$2_{1}^{1}$

$\begin{array}{lll}12509.50 & 12500.50 & \text { P } 9,8\end{array}$

$$
\mathrm{K}^{\prime}=7
$$

$12924.12 \quad 12914.23 \quad$ R 6,6

$12920.82 \quad 12912.37 \quad$ R 7,6

12243

$12232.22 \quad$ P 7,7

$$
\mathrm{K}^{\prime}=6
$$

12582.96

$12571.76 \quad$ R 5,5

$\begin{array}{lll}12218.89 & 12210.37 & \text { P 8,7 }\end{array}$

$12580.28 \quad 12572.04 \quad$ R 5,5

$\begin{array}{lll}12193.33 & 12185.93 & \text { P 9,7 }\end{array}$

12576.67

$12569.63 \quad \mathrm{R} 5,5$

$11955.73 \quad 11945.83 \quad$ P 7,7

$$
\mathrm{K}^{\prime}=5
$$

12244.04

$12234.08 \quad \mathrm{R} 4,4$

$2_{1}^{0}$

$\begin{array}{lrl}11805.15 & 11798.04 & \text { P 10,10 } \\ 11777.01 & 11769.90 & \text { P 11,10 } \\ 11746.08 & 11739.28 & \text { P 12,10 }\end{array}$

$$
K^{\prime}=9
$$

12289.24

$12283.56 \quad$ R 8,8

$12282.39 \quad 12276.32 \quad$ R 9,8

$12274.76 \quad 12268.10 \quad$ R 10,8

11539.92

$11531.31 \quad$ P 9,9

$$
\mathrm{K}^{\prime}=8
$$

11977.69

$11969.08 \quad \mathrm{R} 7,7$

$11511.86 \quad 11504.12 \quad \mathrm{P} 10,9$

$11971.43 \quad 11963.99 \quad \mathrm{R} 8,7$

$11481.55 \quad 11474.74 \quad \mathrm{P} 11,9$

$11968.74 \quad 11963.48 \quad \mathrm{R} 9,7$

$11276.29 \quad 11267.63 \quad$ P 8,8

$$
K^{\prime}=7
$$

11664.77

11656.56 R 6,6

$\begin{array}{lll}11248.87 & 11241.65 & \text { P 9,8 }\end{array}$

11660.47

$11653.42 \quad \mathrm{R} 7,6$

$11221.44 \quad 11214.23 \quad$ p 10,8

11654.63

11648.34 R 8,6

$11017.19 \quad 11009.43 \quad \mathrm{P} 7,7$

$$
K^{\prime}=6
$$

11356.36

11348.92 R 5,5

$10993.25 \quad 10985.8 \quad$ P 8,7

11354.4

11347.55 R 6,5

$10966.24 \quad 10959.98 \quad$ P 9,7

11349.71

$11343.64 \quad \mathrm{R} 7,5$ 


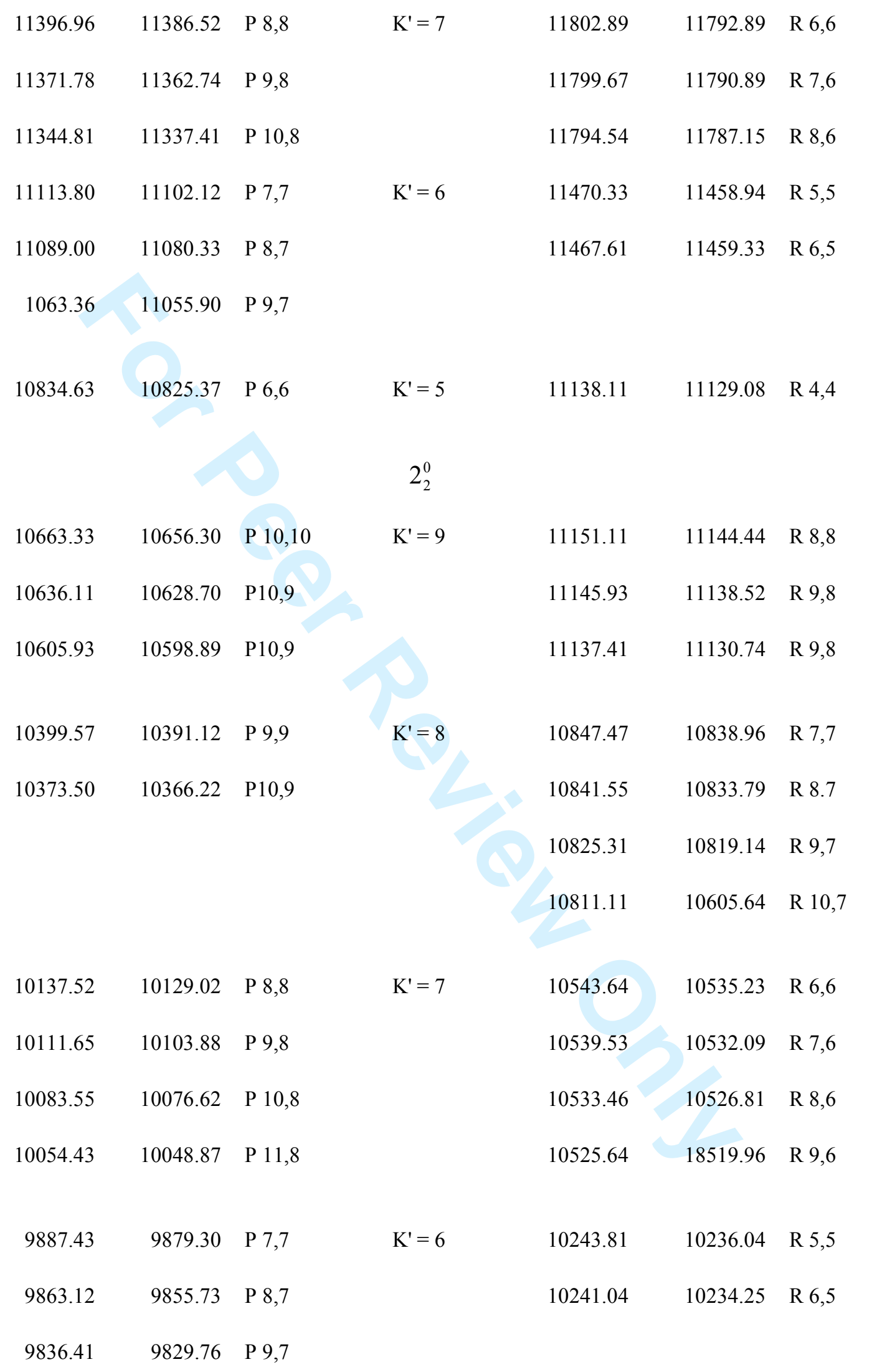




\begin{tabular}{|c|c|c|c|c|c|c|}
\hline 9258.97 & 9250.09 & P 9,9 & $\mathrm{K}^{\prime}=8$ & 9729.20 & 9720.15 & R 7,7 \\
\hline 9282.35 & 9224.21 & P 10,9 & & 9723.29 & 9715.16 & R 8,7 \\
\hline 9009.34 & 9000.09 & P 8,8 & $\mathrm{~K}^{\prime}=7$ & 9435.68 & 9426.80 & R 6,6 \\
\hline 8982.72 & 8974.95 & P 9,8 & & 9431.24 & 9423.11 & R 7,6 \\
\hline 8955.27 & 8947.87 & $\mathrm{P} 10,8$ & & 9425.33 & 9418.67 & R 8,6 \\
\hline 8925.69 & 8919.78 & $\mathrm{P} 11,8$ & & 9417.19 & 9411.28 & R 9,6 \\
\hline 8768.95 & 8760.81 & P 7,7 & $K^{\prime}=6$ & 9145.84 & 9137.71 & R 5,5 \\
\hline 8744.55 & 8737.15 & P 8,7 & & & 9135.68 & R 6,5 \\
\hline 8717.19 & 8711.28 & P 9,7 & & 9151.02 & 9142.88 & R 7,5 \\
\hline 8688.36 & 8682.44 & P 10,7 & & & & \\
\hline 8133.09 & 8123.48 & P 9,9 & $\mathrm{K}^{\prime}=8$ & 8621.81 & 8612.94 & R 7,7 \\
\hline 8104.99 & 8096.86 & P 10,9 & & 8615.90 & 8607.76 & R 8,7 \\
\hline 8076.16 & 8068.02 & P 11,9 & & 8632.16 & 8625.51 & R 9,7 \\
\hline 7891.31 & 7882.44 & P 8,8 & $K^{\prime}=7$ & 8339.37 & 8330.50 & R 6,6 \\
\hline 7865.43 & 7857.30 & P 9,8 & & 8334.94 & 8326.80 & R 7,6 \\
\hline 7837.99 & 7830.59 & P 10,8 & & 8321.63 & 8314.97 & R 8,6 \\
\hline 7808.50 & 7802.59 & P 11,8 & & & & \\
\hline 7661.37 & 7652.50 & P 7,7 & $\mathrm{~K}^{\prime}=6$ & 8060.63 & 8052.50 & R 5,5 \\
\hline 7636.97 & 7629.58 & P 8,7 & & 8076.16 & 8068.02 & R 6,5 \\
\hline 7610.35 & 7603.70 & P 9,7 & & & & \\
\hline
\end{tabular}




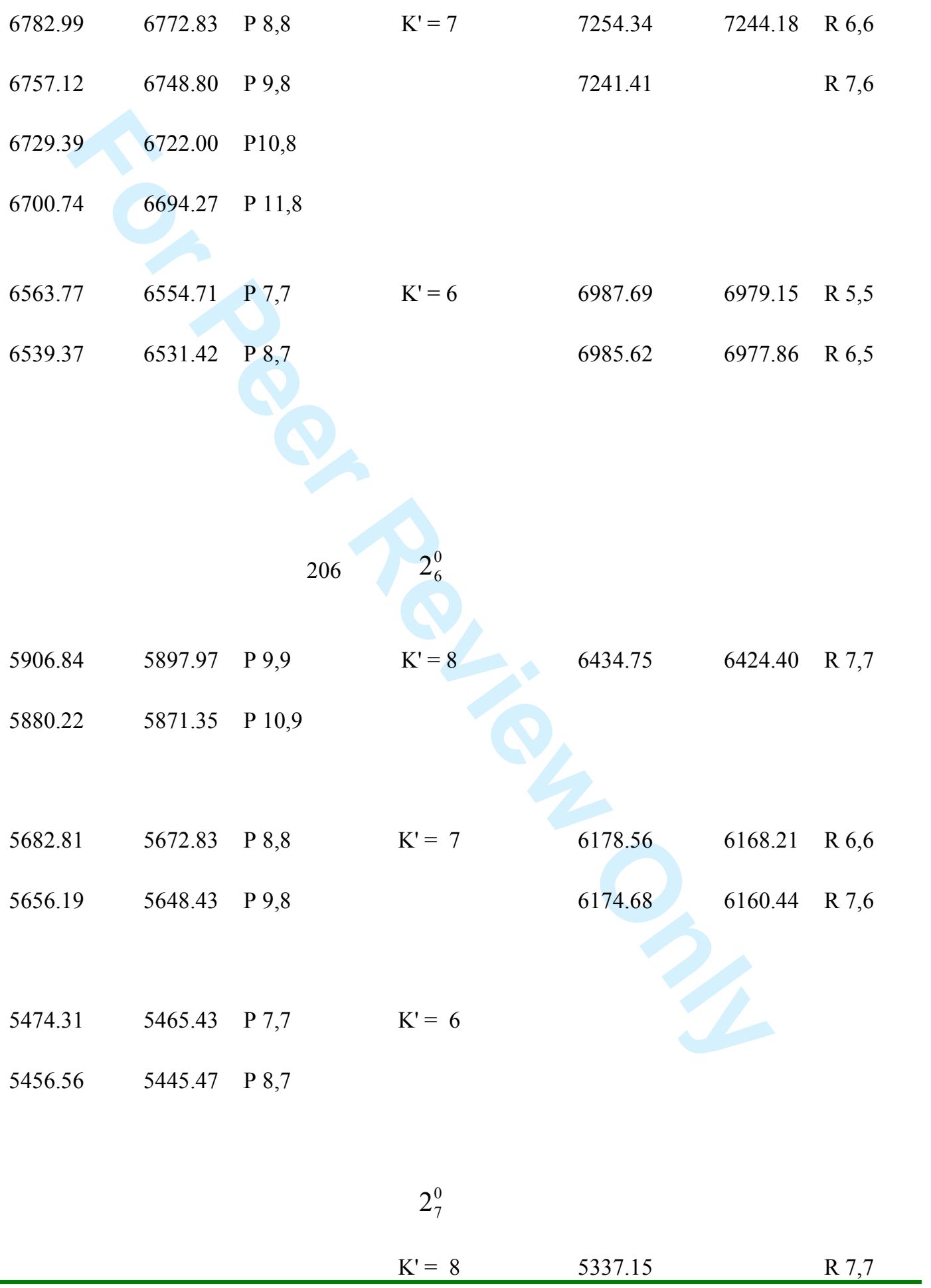


Table 2

Experimentally determined and calculated term values (in cm ${ }^{-1}$ ) for $\mathrm{ND}_{2}\left(\tilde{A}^{2} A_{1}\right), \mathrm{v}_{2}$, $=0$ and 1 vibrational states. The first entry of a pair refers to the $F_{1}$ level and the second to the $\mathrm{F}_{2}$ level. The parameters used for the calculation are given in Table 5, and the calculated effective spin-orbit constants in Table 7.

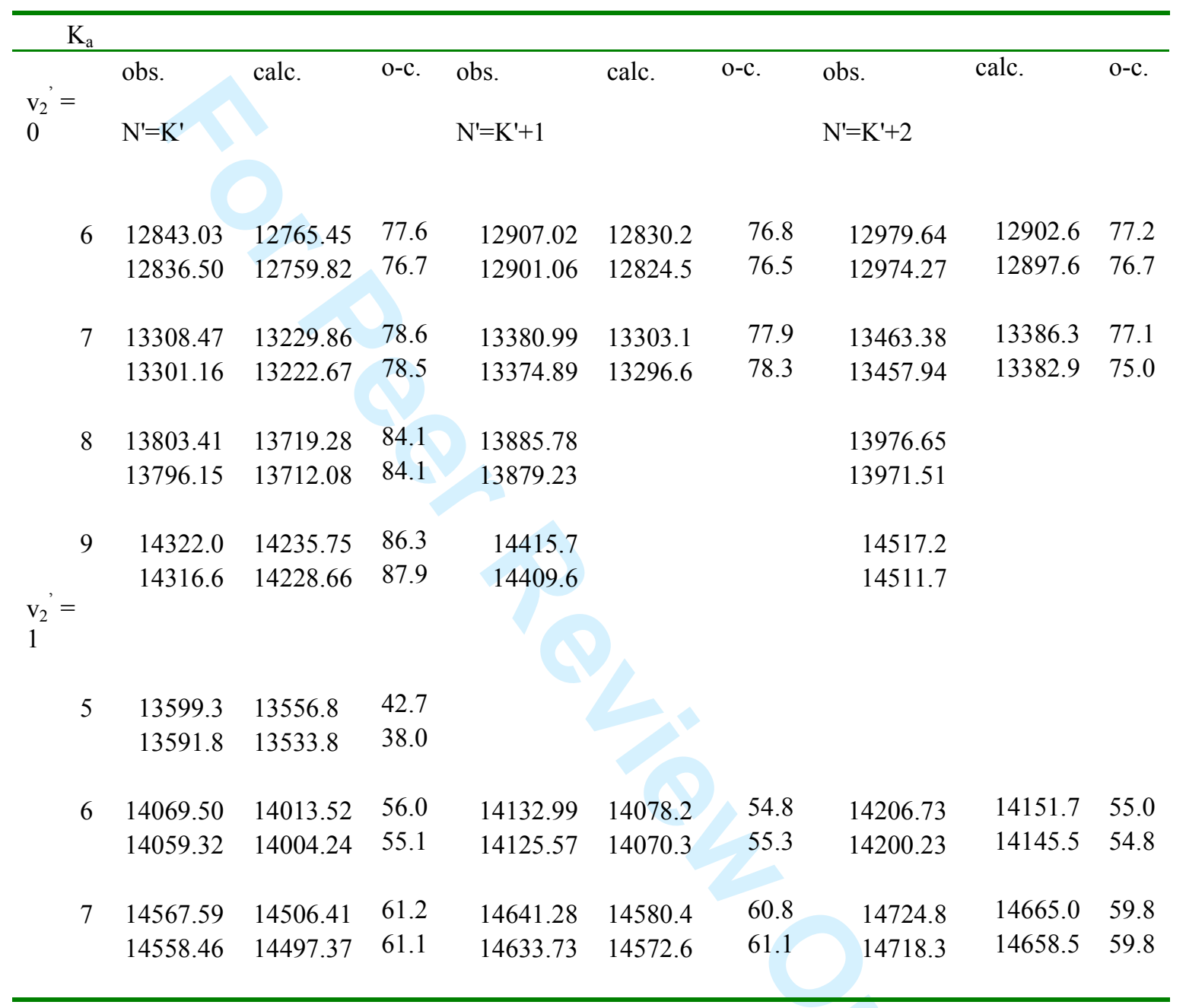




\section{Table 3}

Term values (in $\mathrm{cm}^{-1}$ ) with high values of $\mathrm{K}_{\mathrm{a}}$ for the $0,0,0$ and $0,1,0$ vibrational levels of the $\tilde{X}^{2} B_{1}$ state of ND2. For the $0,0,0$ vibrational state, the levels with values of $\mathrm{K}_{\mathrm{a}}$ $=4$ are taken from Muenchausen et. al. ${ }^{35}$, with those of higher values of $K_{a}$ being derived from the rotational transitions measured by Morino and Kawaguchi ${ }^{36}$. All the term values of the $0,1,0$ vibrational state are taken from from Muenchausen et. al. ${ }^{35}$. In both cases we have averaged the small asymmetry splittings to derive effective symmetric top levels, since the asymmetry splittings of the transitions cannot be resolved at the resolution of the laser induced fluorescence spectra, $0.2 \mathrm{~cm}^{-1}$.

\begin{tabular}{|c|c|c|c|c|c|c|c|}
\hline & & & $v_{2} "=0$ & & & & \\
\hline $\mathrm{N}, \mathrm{K}_{\mathrm{a}}$ & & 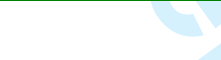 & 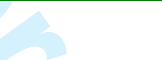 & & & & \\
\hline $\mathrm{N}^{\prime \prime}=\mathrm{K}_{\mathrm{a}}{ }^{\prime \prime}$ & & $N^{\prime \prime}=K_{a} "+1$ & & $N^{\prime \prime}=K_{a}{ }^{\prime \prime}+2$ & & $N "=K_{a} "+3$ & \\
\hline 4,4 & 233.755 & 5,4 & 288.659 & 6,4 & 354.894 & 7,4 & 432.486 \\
\hline & 234.320 & & 289.178 & & 355.354 & & 432.905 \\
\hline 5,5 & 357.219 & 6,5 & 423.109 & 7,5 & 500.183 & 8,5 & 588.481 \\
\hline & 357.937 & & 423.776 & & 500.782 & & 589.032 \\
\hline 6,6 & 505.800 & 7,6 & 582.683 & 8,6 & 670.682 & 9,6 & 769.796 \\
\hline & 506.665 & & 583.493 & & 671.420 & & 770.479 \\
\hline 7,7 & 678.890 & 8,7 & 766.799 & 9,7 & 865.763 & 10,7 & 975.754 \\
\hline & 679.894 & & 767.747 & & 866.637 & & 976.568 \\
\hline 8,8 & 875.775 & 9,8 & 974.789 & 10,8 & 1084.754 & 11,8 & 1205.665 \\
\hline & 876.957 & & 975.869 & & 1085.756 & & 1206.604 \\
\hline 9,9 & 1095.841 & 10,9 & 1205.946 & 11,9 & 1326.942 & 12,9 & 1458.804 \\
\hline & 1097.147 & & 1207.151 & & 1328.068 & & 1459.865 \\
\hline 10,10 & 1338.310 & 10,10 & 1459.537 & 12,10 & 1591.591 & & \\
\hline & 1339.731 & & 1460.859 & & 1592.833 & & \\
\hline
\end{tabular}




\begin{tabular}{|c|c|c|c|c|c|c|c|}
\hline \multicolumn{8}{|c|}{$\mathrm{v}_{2} "=1$} \\
\hline $\mathrm{N}^{\prime \prime}=\mathrm{K}_{\mathrm{a}}{ }^{\prime \prime}$ & & $N "=K_{a} "+1$ & & $\mathrm{~N}^{\prime \prime}=\mathrm{K}_{\mathrm{a}}{ }^{\prime \prime}+2$ & & & $N^{\prime \prime}=K_{a} "+3$ \\
\hline \multirow[t]{2}{*}{4,4} & 1356.079 & 5,4 & 1411.139 & 6,4 & 1477.436 & 7,4 & 1555.115 \\
\hline & 1356.806 & & 1411.759 & & 1477.978 & & 1555.606 \\
\hline \multirow[t]{2}{*}{5,5} & 1486.672 & 6,5 & 1552.625 & 7,5 & 1629.928 & 8,5 & 1718.359 \\
\hline & 1487.578 & & 1553.515 & & 1630.635 & & 1719.004 \\
\hline \multirow[t]{2}{*}{6,6} & 1643.436 & 7,6 & 1720.533 & 8,6 & 1808.717 & 9,6 & 1908.026 \\
\hline & 1644.510 & & 1721.488 & & 1809.583 & & 1908.824 \\
\hline
\end{tabular}


Table 4

Experimentally determined term values (in $\mathrm{cm}^{-1}$ ) for $\mathrm{ND}_{2}$ for the higher vibrational levels of $\tilde{X}^{2} B_{1}$. These were determined using the differences between the wavenumbers of the emission lines of $\mathrm{ND}_{2}$. and those of the new term values of the $\tilde{A}^{2} A_{1}$ state given in Table 2 The precision of new excited state term values relies in turn on the use of the ground state term values given in Table 3 , in combination with the fluorescence spectra terminating in levels with $\mathrm{v}_{2} "=0$ and 1 , which are given in Table 1 . The first entry of a pair refers to $F_{1}$ and the second to $F_{2}$.

\begin{tabular}{|c|c|c|c|}
\hline \multicolumn{4}{|c|}{$\mathrm{v}_{2}=1$} \\
\hline $\begin{array}{l}\mathrm{N}, \mathrm{K}_{\mathrm{a}} \\
\mathrm{K} \equiv \mathrm{K}_{\mathrm{a}}\end{array}$ & $\mathrm{N} "=\mathrm{K} "$ & $N "=K "+1$ & $N "=K "+2$ \\
\hline 7,7 & $\begin{array}{l}1825.8 \\
1827.1\end{array}$ & $\begin{array}{l}1914.1 \\
1915.2\end{array}$ & $\begin{array}{l}2013.4 \\
2014.3\end{array}$ \\
\hline 8,8 & $\begin{array}{l}2032.8 \\
2033.5\end{array}$ & $\begin{array}{l}2131.7 \\
2133.1\end{array}$ & $\begin{array}{l}2242.3 \\
2243.5\end{array}$ \\
\hline 9,9 & $\begin{array}{l}2263.5 \\
2264.8\end{array}$ & $\begin{array}{l}2373.9 \\
2375.1\end{array}$ & $\begin{array}{l}2495.1 \\
2496.8\end{array}$ \\
\hline 10,10 & $\begin{array}{l}2516.9 \\
2518.6\end{array}$ & $\begin{array}{l}2638.7 \\
2639.7\end{array}$ & \\
\hline & & $\mathrm{v}_{2}=2$ & \\
\hline 5,5 & $\begin{array}{l}2599.2 \\
2600.5\end{array}$ & $\begin{array}{l}2666.0 \\
2666.8\end{array}$ & 2746.0 \\
\hline 6,6 & $\begin{array}{l}2764.8 \\
2765.9\end{array}$ & $\begin{array}{l}2841.5 \\
2842.8\end{array}$ & $\begin{array}{l}2929.9 \\
2931.1\end{array}$ \\
\hline 7,7 & $\begin{array}{l}2955.9 \\
2957.2\end{array}$ & $\begin{array}{l}3044.2 \\
3045.4\end{array}$ & $\begin{array}{l}3151.3 \\
3152.4\end{array}$ \\
\hline 8,8 & $\begin{array}{l}3183.7 \\
3185.0\end{array}$ & $\begin{array}{l}3280.1 \\
3280.1\end{array}$ & \\
\hline
\end{tabular}




$$
\mathrm{v}_{2}=3
$$

\begin{tabular}{|c|c|c|c|}
\hline \multirow[t]{2}{*}{5,5} & 3697.2 & & 3828.6 \\
\hline & 3698.8 & 3765.4 & 3831.4 \\
\hline \multirow[t]{2}{*}{6,6} & 3872.8 & 3949.8 & 4038.1 \\
\hline & 3874.4 & 3951.8 & 4039.3 \\
\hline \multirow[t]{2}{*}{7,7} & 4074.2 & 4162.5 & \\
\hline & 4066.8 & 4164.1 & \\
\hline \multirow[t]{2}{*}{8,8} & 4299.1 & 4398.3 & 4508.1 \\
\hline & 4301.1 & 4399.9 & 4510.1 \\
\hline \multirow[t]{2}{*}{9,9} & 4544.4 & 4603.4 & \\
\hline & 4546.1 & 4655.0 & \\
\hline
\end{tabular}

$\begin{array}{llll}5,5 & 4782.4 & 4830.9 & 4941.5 \\ 4784.0 & 4833.0 & \end{array}$

$\begin{array}{llll}6,6 & 4969.1 & 5046.1 & 5141.8\end{array}$

$4970.7 \quad 5048.1 \quad 5143.0$

$\begin{array}{llll}7,7 & 5181.6 & 5269.9 & 5344.5 \\ & 5183.2 & 5271.5 & 5346.0 \\ & & & \\ 8,8 & 5417.2 & 5515.6 & 5625.4 \\ & 5418.7 & 5517.6 & 5627.3\end{array}$

$\begin{array}{llll}9,9 & 5670.3 & 5780.8 & 5900.5 \\ 5672.7 & 5782.4 & 5903.5\end{array}$

$$
\mathrm{v}_{2}=5
$$

\begin{tabular}{llll}
5,5 & 5909.4 & 6001.1 & 6022.5 \\
\hline
\end{tabular}




\begin{tabular}{|c|c|c|c|}
\hline & 5913.0 & 6003.5 & \\
\hline 6,6 & $\begin{array}{l}6054.1 \\
6057.0\end{array}$ & 6139.6 & 6254.8 \\
\hline 7,7 & $\begin{array}{l}6279.4 \\
6281.7\end{array}$ & $\begin{array}{l}6367.7 \\
6369.6\end{array}$ & \\
\hline 8,8 & $\begin{array}{l}6525.5 \\
6528.3\end{array}$ & $\begin{array}{l}6623.9 \\
6626.1\end{array}$ & $\begin{array}{l}6734.0 \\
6735.9\end{array}$ \\
\hline 9,9 & $\begin{array}{l}6788.4 \\
6790.4\end{array}$ & $\begin{array}{l}6898.5 \\
6900.3\end{array}$ & \\
\hline & & & \\
\hline 6,6 & $\begin{array}{l}7129.9 \\
7133.0\end{array}$ & $\begin{array}{l}7206.3 \\
7214.4\end{array}$ & \\
\hline 7,7 & $\begin{array}{l}7368.7 \\
7371.8\end{array}$ & & \\
\hline 8,8 & $\begin{array}{l}7625.7 \\
7628.3\end{array}$ & $\begin{array}{l}7724.8 \\
7726.5\end{array}$ & \\
\hline 9,9 & $\begin{array}{l}7896.6 \\
7898.2\end{array}$ & $\begin{array}{l}8005.6 \\
8007.9\end{array}$ & \\
\hline
\end{tabular}


Table 5

Parameters used to model the rotational structure of the $\tilde{X}^{2} B_{1}$ and $\tilde{A}^{2} A_{1}$ states of $\mathrm{ND}_{2}$ and the Renner-Teller coupling between them. Most of the parameters are identical to those used to calculate the spectrum of $\mathrm{NH}_{2}$ (refs. 33 and 34). The parameters which differ from those used for $\mathrm{NH}_{2}$, are those in which the parameter used for $\mathrm{NH}_{2}$ was not isotopically independent. The parameters which required to be changed are the asymmetric stretching frequencies $\omega_{3}$ for the $\tilde{A}^{2} A_{1}$ and the $\tilde{X}^{2} B_{1}$ states, and the value of the parameter for the variation of $\left\langle L_{z}\right\rangle, g_{K}$.

$$
\tilde{A}^{2} A_{1} \text { state }
$$

Bond length variation

$r(\rho=0)$

$a$, coefficient of $\rho^{2}$

$b$, coefficient of $\tan ^{2} \rho$

$f_{0}$

$m$

$H$

$\mathrm{k}_{4}$

$\mathrm{c}_{1}$

$f_{r r}^{s}$

$\mathrm{x}_{1}$

$\omega_{3}$

$\mathrm{x}_{3}$

$\delta_{11}$

$\delta_{21}$

$\delta k_{41}$

$\tilde{X}^{2} B_{1}$ state

Bond length variation

$r(\rho=0)$

$a$, coefficient of $\rho^{2}$

$b$, coefficient of $\tan ^{2} \rho$

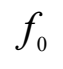

$m$

H

$\mathrm{k}_{4}$

$\mathrm{c}_{1}$

$f_{r r}^{s}$

$\mathrm{x}_{1}$

$\omega_{3}$

$\mathrm{x}_{3}$

$\delta_{11}$

$\delta_{21}$

$\delta k_{41}$

$\delta k_{42}$

\begin{tabular}{|c|c|}
\hline $13249.59 \pm 16$ & $\begin{array}{l}0.996 \AA \\
0.000 \AA \\
0.1063 \AA \\
\mathrm{cm}^{-1} \mathrm{rad}^{-2}\end{array}$ \\
\hline 35.814 & $\operatorname{deg}$ \\
\hline 729.9 & $\mathrm{~cm}^{-1}$ \\
\hline$-0.35 \pm 50$ & $\mathrm{~cm}^{-1} \mathrm{rad}^{-4}$ \\
\hline$-53.01 \pm 5$ & $\mathrm{~cm}^{-1}$ \\
\hline 8.9093 & $\mathrm{aJ} \AA^{2}$ \\
\hline-0.0025 & \\
\hline 2536.7 & $\mathrm{~cm}^{-1}$ \\
\hline-0.0033 & \\
\hline 17.07 & $\mathrm{~cm}^{-1}$ \\
\hline 0.00 & $\mathrm{~cm}^{-1}$ \\
\hline $131.94 \pm 130$ & $\mathrm{~cm}^{-1} \mathrm{rad}^{-4}$ \\
\hline & $0.996 \AA$ \\
\hline & $0.0146 \AA$ \\
\hline & $0.0185 \AA$ \\
\hline $33596.53 \pm 13$ & $\mathrm{~cm}^{-1} \mathrm{rad}^{-2}$ \\
\hline 77.6 & deg \\
\hline 11773.67 & $\mathrm{~cm}^{-1}$ \\
\hline $374.1 \pm 40$ & $\mathrm{~cm}^{-1} \mathrm{rad}^{-4}$ \\
\hline $154.22 \pm 40$ & $\mathrm{~cm}^{-1}$ \\
\hline 8.3955 & $\mathrm{aJ} \AA^{2}$ \\
\hline-0.01440 & \\
\hline 2391.1 & $\mathrm{~cm}^{-1}$ \\
\hline-0.0093 & \\
\hline $505.55 \pm 100$ & $\mathrm{~cm}^{-1}$ \\
\hline$-781.93 \pm 250$ & $\mathrm{~cm}^{-1}$ \\
\hline $765.98 \pm 400$ & $\mathrm{~cm}^{-1} \mathrm{rad}^{-4}$ \\
\hline$-793.14 \pm 300$ & $\mathrm{~cm}^{-1} \mathrm{rad}^{-4}$ \\
\hline
\end{tabular}


Spin-orbit coupling parameter, $A^{\text {SO }} \quad 61.6 \quad \mathrm{~cm}^{-1}$

Variation of $\left\langle L_{z}\right\rangle, g_{K} \quad 3.36 \quad \mathrm{~cm}^{-1}$

The errors on the parameters were those derived from the final least squares fitting of the $\mathrm{NH}_{2}$ spectrum. 
Table 6

Observed and calculated molecular parameters (in $\mathrm{cm}^{-1}$ ) for the vibronic levels of the $\tilde{X}^{2} B_{1}$ and the $\tilde{A}^{2} A_{1}$ states of $\mathrm{ND}_{2}$ which were analysed by Dressler and Ramsay $(\mathrm{D} \& \mathrm{R})^{5}$. The average vibronic origins are denoted by $\mathrm{T}$, and the vibronic spin orbit coupling constant by $A_{v, K}^{S O} \cdot A_{v, K}^{S O}$ is identically zero for levels having $\mathrm{K}=0$. Since the stretch-bender model incorporates the effects of Fermi resonance, these may be observed for some of the higher vibrational levels of $\mathrm{ND}_{2}$, and are indicated by the inclusion of $v_{1}$.

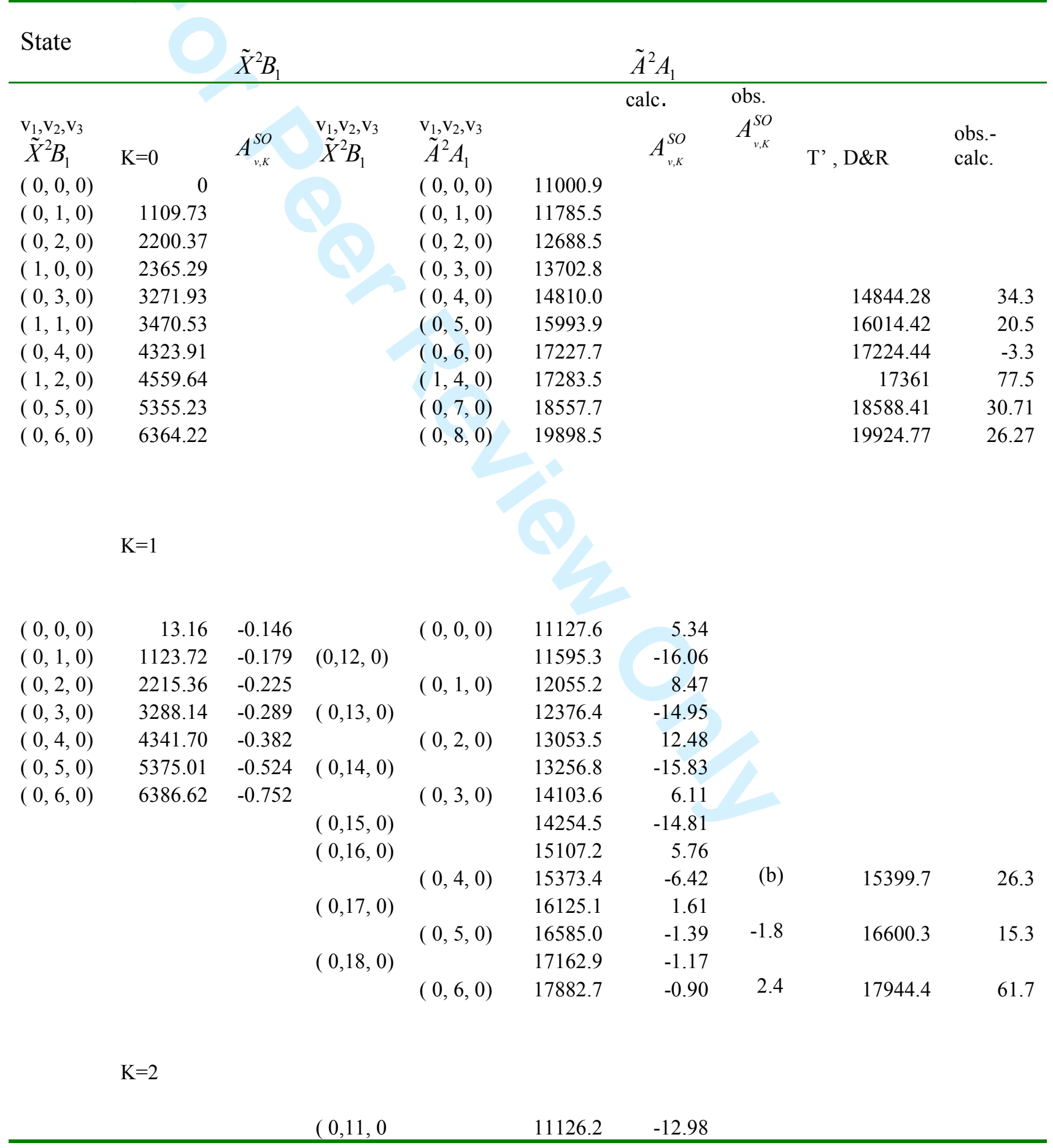




\begin{tabular}{|c|c|c|c|c|c|c|c|c|c|}
\hline$(0,0,0)$ & 52.57 & -0.290 & & $(0,0,0)$ & 11338.1 & 5.47 & & & \\
\hline$(0,1,0)$ & 1165.59 & -0.356 & $(0,12,0)$ & & 11946.5 & -13.21 & & & \\
\hline$(0,2,0)$ & 2260.20 & -0.444 & & $(0,1,0)$ & 12356.9 & 9.18 & & & \\
\hline$(0,3,0)$ & 3336.66 & -0.569 & $(0,13,0)$ & & 12819.8 & -13.79 & & & \\
\hline$(0,4,0)$ & 4394.75 & -0.747 & & $(0,2,0)$ & 13425.5 & 12.52 & & & \\
\hline$(0,5,0)$ & 5433.84 & -1.012 & $(0,14,0)$ & & 13752.5 & -14.65 & & & \\
\hline \multirow[t]{11}{*}{$(0,6,0)$} & 6452.95 & -1.428 & & $(0,3,0)$ & 14522.5 & 12.90 & & & \\
\hline & & & $(0,15,0)$ & & 14772.0 & -11.42 & & & \\
\hline & & & $(0,16,0)$ & & 15581.7 & 6.73 & & & \\
\hline & & & & $(0,4,0)$ & 15920.5 & -6.36 & $\mathrm{c}$ & 15936.1 & 15.6 \\
\hline & & & $(0,17,0)$ & & 166201 & 9.81 & & & \\
\hline & & & & $(0,5,0)$ & 17147.2 & -1.79 & $\mathrm{c}$ & 17141.1 & -6.1 \\
\hline & & & $(0,18,0)$ & & 18216.0 & 5.00 & & & \\
\hline & & & & $(0,6,0)$ & 18469.1 & 3.12 & d & 18485.6 & 16.5 \\
\hline & & & $(0,20,0)^{\mathrm{a}}$ & $(0,7,0)$ & 19759.0 & 9.36 & & & \\
\hline & & & $(0,20,0)^{\mathrm{a}}$ & $(0,7,0)$ & 19895.9 & -11.00 & d & 19857.5 & -38.4 \\
\hline & $K=3$ & & & & & & & & \\
\hline$(0,0,0)$ & 118.04 & -0.431 & & $(0,0,0)$ & 11613.1 & 6.37 & & & \\
\hline$(0,1,0)$ & 1235.06 & -0.527 & $(0,12,0)$ & & 12297.2 & -12.34 & & & \\
\hline$(0,2,0)$ & 2334.50 & -0.654 & & $(0,1,0)$ & 12705.2 & 9.99 & & & \\
\hline$(0,3,0)$ & 3416.83 & -0.831 & $(0,13,0)$ & & 13231.0 & -13.69 & & & \\
\hline$(0,4,0)$ & 4482.14 & -1.08 & & $(0,2,0)$ & 13848.0 & 6.12 & & & \\
\hline$(0,5,0)$ & 5530.26 & -1.441 & $(0,14,0)$ & & 14213.9 & -15.29 & & & \\
\hline \multirow[t]{6}{*}{$(0,6,0)$} & 6560.82 & -1.983 & & $(0,3,0)$ & 14972.1 & 12.65 & & & \\
\hline & & & $(0,15,0)$ & & 15274.2 & -13.59 & & & \\
\hline & & & $(0,16,0)$ & & 16067.8 & 4.75 & & & \\
\hline & & & & $(0,4,0)$ & 16450.8 & -4.63 & & & \\
\hline & & & $(0,17,0)$ & & 17139.2 & -0.70 & & & \\
\hline & & & & $(0,5,0)$ & 17748.3 & -3.18 & & 17838 & 89.666 \\
\hline
\end{tabular}

a, Strong calculated resonant interaction between the two nearly degenerate states, ${ }^{2} B_{1} v_{2}=20$ and ${ }^{2} A_{1} v_{2}=7$, leads to the predicted 50:50 mixing.

b Perturbed

c Unresolved splitting

d Inverted splitting, small. 
Table 7

Observed and calculated molecular parameters (in $\mathrm{cm}^{-1)}$ for the vibronic levels of the $\tilde{X}^{2} B_{1}$ and the $\tilde{A}^{2} A_{1}$ states of $\mathrm{ND}_{2}$ which are measured in the high angular momentum emission spectrum. The average vibronic origins are denoted by $\mathrm{T}$, and the vibronic spin orbit coupling constant by $A_{v, K}^{S O}$.

\begin{tabular}{|c|c|c|c|c|c|c|c|c|c|c|}
\hline State & $K=K_{a}$ & $\tilde{X}^{2} B_{1}$ & & & & $\tilde{A}^{2} A_{1}$ & & & & \\
\hline $\begin{array}{l}\left(\mathrm{v}_{1}, \mathrm{v}_{2}, \mathrm{v}_{3}\right) \\
\tilde{X}^{2} B_{1}\end{array}$ & $\begin{array}{l}\mathrm{T} ” \\
\mathrm{~K}=5\end{array}$ & $A_{v, K}^{S O}$ & 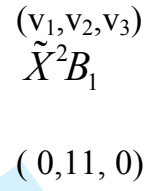 & $\begin{array}{l}\left(\mathrm{v}_{1}, \mathrm{v}_{2}, \mathrm{v}_{3}\right) \\
\tilde{A}^{2} A_{1}\end{array}$ & $\begin{array}{l}\text { T' } \\
12028.5\end{array}$ & $\begin{array}{l}A_{v, K}^{S O} \\
-9.91\end{array}$ & T' obs. & $\begin{array}{l}\text { obs- } \\
\text { calc }\end{array}$ & $\begin{array}{l}\bar{B}^{\text {a }} \\
\text { obs }\end{array}$ & $\begin{array}{l}\bar{B}^{\text {a }} \\
\text { calc }\end{array}$ \\
\hline$(0,0,0)$ & 325.82 & -0.70 & & $(0,0,0)$ & 12320.5 & 7.67 & & & & \\
\hline \multirow[t]{2}{*}{$(0,1,0)$} & 1454.95 & -0.85 & $(0,12,0)$ & & 13031.8 & -11.26 & & & & \\
\hline & & & & & 13524.0 & & & & & \\
\hline$(0,2,0)$ & 2568.73 & -1.04 & $(0,13,0)$ & & & 10.26 & & & & \\
\hline$(0,3,0)$ & 3668.20 & -1.29 & & & 14052.0 & -12.56 & & & & \\
\hline$(0,4,0)$ & 4754.11 & -1.63 & & & & & & & & \\
\hline$(0,5,0)$ & 5827.13 & -2.09 & & $(0,2,0)$ & 14738.8 & -12.03 & & & & \\
\hline \multirow[t]{3}{*}{$(0,6,0)$} & 6887.95 & -2.72 & & & & & & & & \\
\hline & $\mathrm{K}=6$ & & & & & & & & & \\
\hline & & & $(0,11,0)$ & & 12374.5 & -9.24 & & & & \\
\hline$(0,0,0)$ & 467.28 & -0.82 & & $(0,0,0)$ & 12740.6 & 7.79 & 12812.2 & 71.6 & 4.59 & 4.63 \\
\hline$(0,1,0)$ & 1604.17 & -0.99 & $(0,12,0)$ & & 13414.5 & -10.52 & & & & \\
\hline$(0,2,0)$ & 2726.98 & -1.20 & & $(0,1,0)$ & 13984.1 & 10.20 & 14008.9 & 24.8 & 4.65 & 4.66 \\
\hline$(0,3,0)$ & 3837.01 & -1.48 & $(0,13,0)$ & & 14467.5 & -11.66 & & & & \\
\hline$(0,4,0)$ & 4935.31 & -1.84 & & $(0,2,0)$ & 15238.2 & 10.93 & & & & \\
\hline$(0,5,0)$ & 6022.82 & -2.32 & & & & & & & & \\
\hline \multirow[t]{2}{*}{$(0,6,0)$} & 7100.53 & -2.95 & & & & & & & & \\
\hline & $\mathrm{K}=7$ & & & & & & & & & \\
\hline$(0,0,0)$ & 633.04 & -0.94 & $(0,11,0)$ & & 12736.7 & -8.58 & & & & \\
\hline$(0,1,0)$ & 1778.56 & -1.12 & & $(0,0,0)$ & 13194.8 & 7.81 & 13272.8 & 78.0 & 4.57 & 4.67 \\
\hline$(0,2,0)$ & 2911.28 & -1.35 & $(0,12,0)$ & & 13807.2 & -9.75 & & & & \\
\hline$(0,3,0)$ & 4032.77 & -1.64 & & $(0,1,0)$ & 14472.9 & 9.83 & 14534.0 & 61.1 & 4.66 & 4.7 \\
\hline$(0,4,0)$ & 5144.25 & -2.01 & $(0,13,0)$ & & 14888.3 & -10.72 & & & & \\
\hline$(0,5,0)$ & 6246.86 & -2.49 & & $(0,2,0)$ & 15753.4 & 9.46 & & & & \\
\hline$(0,6,0)$ & 7341.74 & -3.10 & & & & & & & & \\
\hline
\end{tabular}




\begin{tabular}{|c|c|c|c|c|c|c|c|c|c|c|}
\hline & $\mathrm{K}=8$ & & & & & & & & & \\
\hline & & & $(0,11,0)$ & & 13113.8 & -7.95 & & & & \\
\hline$(0,0,0)$ & 822.46 & -1.05 & & $(0,0,0)$ & 13679.7 & 768 & 13763.0 & 85.8 & 4.6 & 4.71 \\
\hline$(0,1,0)$ & 1977.32 & -1.25 & $(0,12,0)$ & & 14202.9 & -8.98 & & & & \\
\hline$(0,2,0)$ & 3120.58 & -1.48 & & $(0,1,0)$ & 14987.0 & 9.30 & & & & \\
\hline$(0,3,0)$ & 4354.08 & -1.78 & $(0,13,0)$ & & 15315.3 & -9.68 & & & & \\
\hline$(0,4,0)$ & 5379.17 & -2.15 & & $(0,2,0)$ & 16282.3 & 5.83 & & & & \\
\hline$(0,5,0)$ & 6497.08 & -2.62 & & & & & & & & \\
\hline \multirow[t]{2}{*}{$(0,6,0)$} & 7608.99 & -3.20 & & & & & & & & \\
\hline & $\mathrm{K}=9$ & & & & & & & & & \\
\hline$(0,0,0)$ & 1034.90 & -1.16 & & $(0,0,0)$ & 14191.5 & 7.49 & 14277.3 & 85.0 & 4.67 & 4.73 \\
\hline$(0,1,0)$ & 2199.62 & -1.36 & $(0,12,0)$ & & 14621.1 & -8.10 & & & & \\
\hline$(0,2,0)$ & 3353.72 & -1.60 & & $(0,1,0)$ & 15523.1 & 8.58 & & & & \\
\hline$(0,3,0)$ & 4499.53 & -1.89 & $(0,13,0)$ & & 15747.1 & -8.28 & & & & \\
\hline$(0,4,0)$ & 5638.36 & -2.26 & $(0,14,0)$ & & 16802.6 & -1.41 & & & & \\
\hline$(0,5,0)$ & 6771.43 & -2.71 & & $(0,2,0)$ & 16989.7 & -1.77 & & & & \\
\hline \multirow[t]{3}{*}{$(0,6,0)$} & 7899.90 & -3.25 & & & & & & & & \\
\hline & $\mathrm{K}=10$ & & & & & & & & & \\
\hline & & & $(0,11,0)$ & & 14202.1 & -7.6 & & & & \\
\hline$(0,0,0)$ & 1272.36 & -1.26 & & $(0,0,0)$ & 14721.99 & 7.19 & & & & \\
\hline$(0,1,0)$ & 2454.77 & -1.48 & $(0,12,0)$ & & 15390.62 & -8.46 & & & & \\
\hline$(0,2,0)$ & 3632.17 & -1.75 & & $(0,1,0)$ & 16083.47 & 8.55 & & & & \\
\hline$(0,3,0)$ & 4805.86 & -2.07 & $(0,13,0)$ & & 16587.71 & -9.26 & & & & \\
\hline$(0,4,0)$ & 5977.09 & -2.47 & & $(0,2,0)$ & 17475.51 & 9.59 & & & & \\
\hline$(0,5,0)$ & 7147.11 & -2.94 & $(0,14,0)$ & & 17801.63 & -9.69 & & & & \\
\hline$(0,6,0)$ & 8317.12 & -3.5 & & & (1) & & & & & \\
\hline
\end{tabular}

a $\quad \bar{B}=\frac{1}{2}(B+C)$ 
Figures and Figure Captions

Figure 1
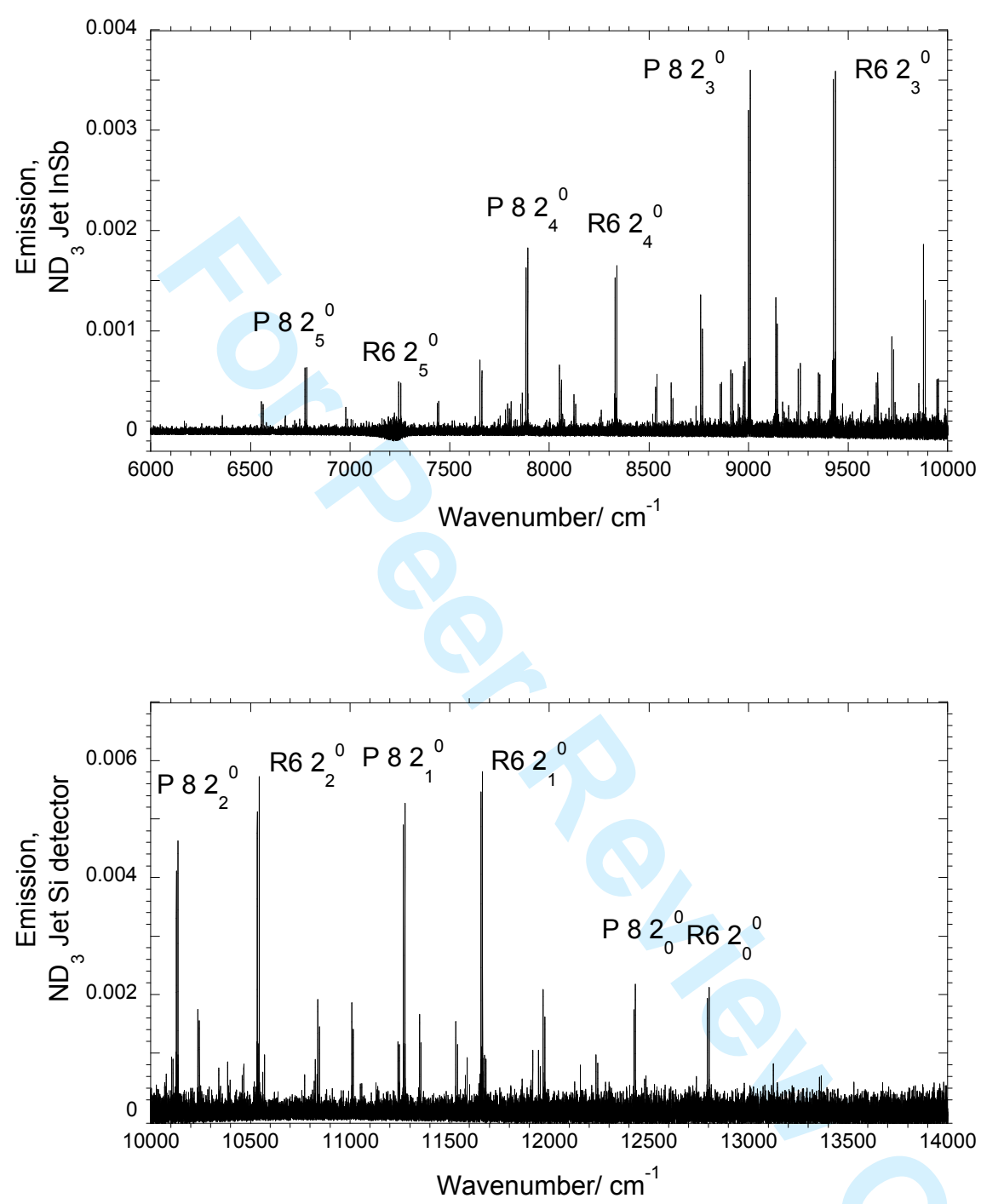

A survey of the entire product emission spectrum of $\mathrm{ND}_{2}\left(\tilde{A}^{2} A_{1}\right)$ from the

photodissociation of jet cooled $\mathrm{ND}_{3}$. 1(a) $6000-10,000 \mathrm{~cm}^{-1}$, InSb detector: 1(b) 10,000 to $14,000 \mathrm{~cm}^{-1}$, a Si avalanche detector. This spectrum is dominated by the strong ${ }^{\mathrm{R}} \mathrm{R} 6$ and ${ }^{\mathrm{P}} \mathrm{P} 8$ transitions with $\mathrm{N}=\mathrm{K}$, resulting from the production of $\mathrm{ND}_{2}$ in the 0,0,0 level of the $\tilde{A}^{2} A_{1}$ state predominantly with $\mathrm{K}_{\mathrm{a}}=7$. 
Figure 2

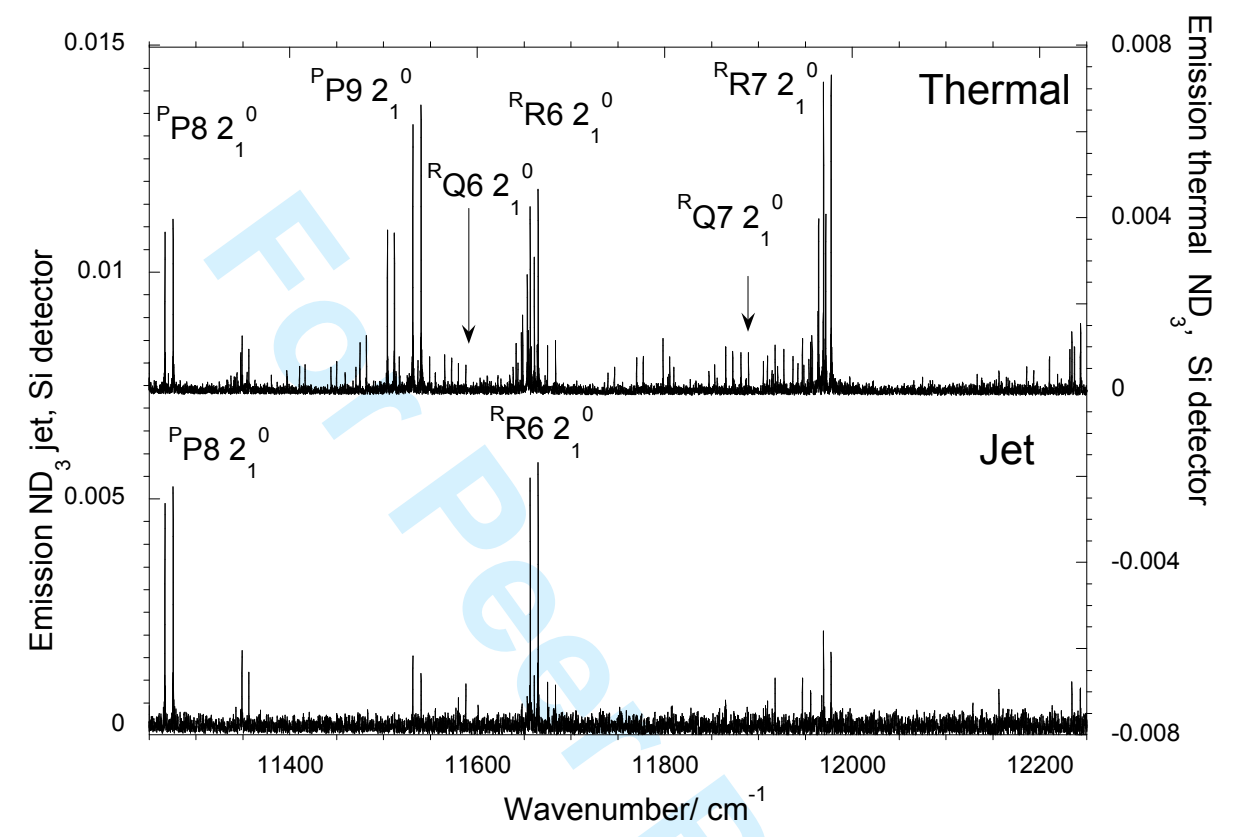

An expanded section of the spectrum showing the doublet structure of the transitions, and also demonstrating the more complex patterns seen ${ }^{\mathrm{R}} \mathrm{R}$ and ${ }^{\mathrm{P}} \mathrm{P}$ branches in the spectra produced from the photodissociation of room temperature $\mathrm{ND}_{3}$. In this spectrum the strongly degraded structure of the ${ }^{\mathrm{P}} \mathrm{P}$ branches contrasts with the compact structure of the ${ }^{\mathrm{R}} \mathrm{R}$ branches. 
Figure 3
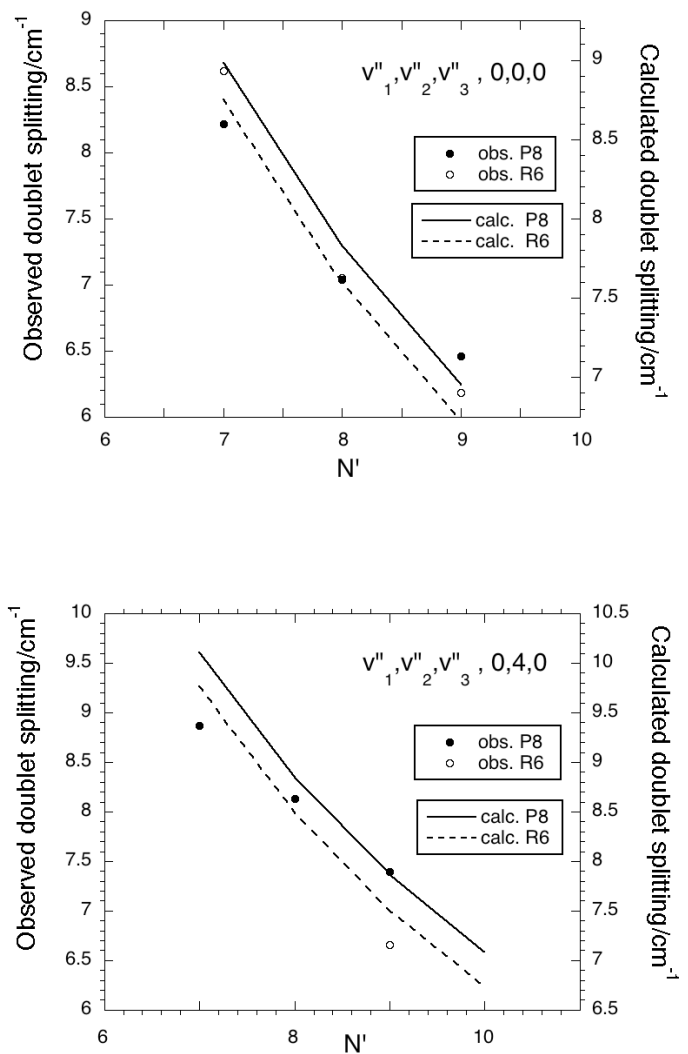

Plots of the effects of spin uncoupling on the separation of the $F_{1}-F_{2}$ doublets of the ${ }^{\mathrm{R}} \mathrm{R} 6$ and ${ }^{\mathrm{P}} \mathrm{P} 8$ transitions in (a) the $2{ }_{0}^{0}$ and (b) the $2{ }_{4}^{0}$ bands. The experimental splitting is compared with that calculated using the stretch-bender Renner-Teller Hamiltonian ${ }^{31,32}$ using the same parameters as for $\mathrm{NH}_{2}$, see Table 5. It may be seen that the doublet splittings are significantly greater when $\mathrm{v}_{2}$ " is increased from 0 to 4 , but the errors in the experimental measurements are larger than the calculated increase in the splitting from ${ }^{\mathrm{R}} \mathrm{R}$ to ${ }^{\mathrm{P}} \mathrm{P}$ components of transitions sharing a pair of $\mathrm{F}_{1}$ and $\mathrm{F}_{2}$ with the same values of $\mathrm{N}^{\prime}$ and $\mathrm{K}_{\mathrm{a}}$ '. 
Figure 4

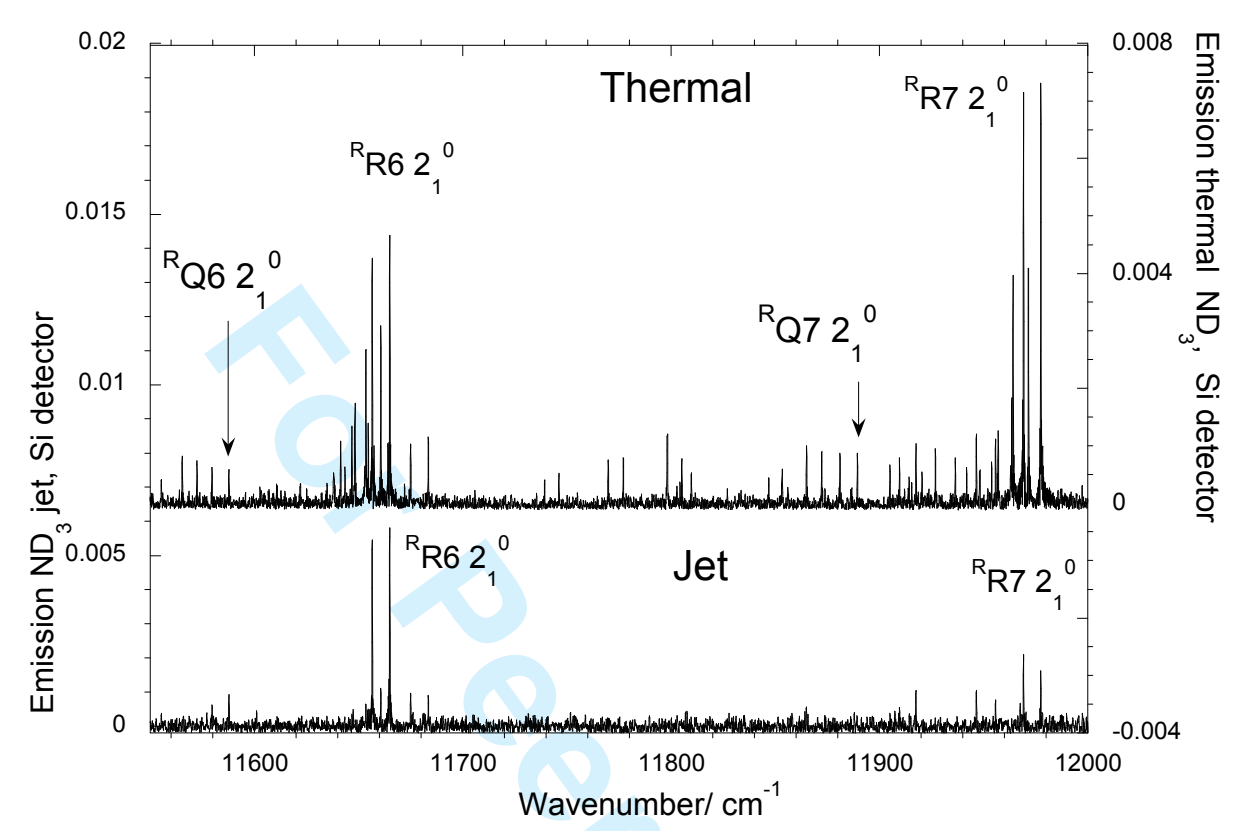

An expanded section of the spectrum shown in Figure 2. The ${ }^{\mathrm{R}} \mathrm{Q} 7$ and ${ }^{\mathrm{R}} \mathrm{Q} 6$ transitions are located on the red side of the equivalent ${ }^{\mathrm{R}} \mathrm{R} 7$ and ${ }^{\mathrm{R}} \mathrm{R} 6$ branches.

Although these Q branches are weak and difficult to detect in the jet cooled spectrum, near the band centre when $K_{a}{ }^{\prime}=0$ and 1 , the line strength of the ${ }^{\mathrm{R}} \mathrm{Q}$ and equivalent ${ }^{\mathrm{P}}$ $\mathrm{Q}$ branches is much greater than that of the ${ }^{\mathrm{R}} \mathrm{R}$ and ${ }^{\mathrm{P}} \mathrm{P}$ transitions. However it has been difficult to find evidence for the occurrence of these branches with $\mathrm{K}_{\mathrm{a}}{ }^{\prime}=0$ and 1 in the emission spectrum of $\mathrm{ND}_{2}$ obtained from the photodissociation of $\mathrm{ND}_{3}$. 
Figure 5

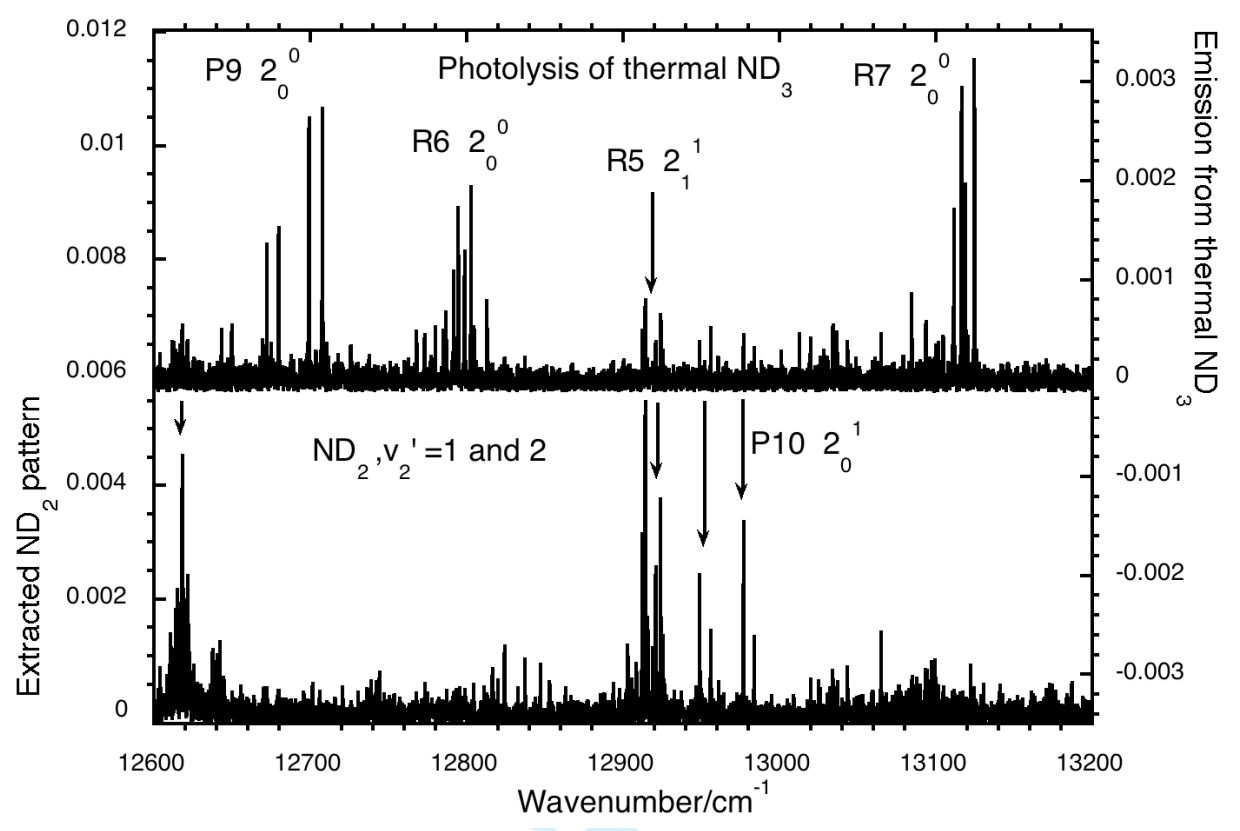

A comparison of the spectrum produced from the photodissociation of room

temperature $\mathrm{ND}_{3}$ with an "extracted" spectrum of emission from $\mathrm{ND}_{2}\left(\tilde{A}^{2} A_{1}\right)$ in which only the $\mathrm{v}_{2}^{\prime}=1$ and 2 states are populated. This method of extracting product state emission patterns

has been described by Reid, Loomis and Leone ${ }^{30}$. An example of this is shown in Fig 5 of [30] in which an overview spectrum showing this use of this extraction method to aid the comparison of these, and related spectra, is presented. In the present spectra it may be seen that several of the weak sub-band structures in the $\mathrm{ND}_{3}$ spectrum are clearly identified as belonging to hot bands having $\mathrm{v}_{2}{ }^{\prime}=1$ and 2 . The most obvious patterns are indicated by arrows. The strongest transitions originating from $\mathrm{v}_{2}{ }^{\prime}=1$ are identified. 